\title{
Understanding extreme quasar optical variability with CRTS - I. Major AGN flares
}

\author{
Matthew J. Graham, ${ }^{1 \star}$ S. G. Djorgovski, ${ }^{1}$ Andrew J. Drake, ${ }^{1}$ Daniel Stern, ${ }^{2}$ \\ Ashish A. Mahabal, ${ }^{1}$ Eilat Glikman, ${ }^{3}$ Steve Larson ${ }^{4}$ and Eric Christensen ${ }^{4}$ \\ ${ }^{1}$ California Institute of Technology, 1200 E. California Blvd, Pasadena, CA 91125, USA \\ ${ }^{2}$ Jet Propulsion Laboratory, California Institute of Technology, 4800 Oak Grove Drive, Pasadena, CA 91109, USA \\ ${ }^{3}$ Department of Physics, Middlebury College, Middlebury, VT 05753, USA \\ ${ }^{4}$ Department of Planetary Sciences, University of Arizona, Lunar and Planetary Lab, Tucson, AZ 85721, USA
}

Accepted 2017 June 8. Received 2017 June 6; in original form 2017 March 8

\begin{abstract}
There is a large degree of variety in the optical variability of quasars and it is unclear whether this is all attributable to a single (set of) physical mechanism(s). We present the results of a systematic search for major flares in active galactic nucleus (AGN) in the Catalina Real-time Transient Survey as part of a broader study into extreme quasar variability. Such flares are defined in a quantitative manner as being atop of the normal, stochastic variability of quasars. We have identified 51 events from over 900000 known quasars and high-probability quasar candidates, typically lasting $900 \mathrm{~d}$ and with a median peak amplitude of $\Delta m=1.25$ mag. Characterizing the flare profile with a Weibull distribution, we find that nine of the sources are well described by a single-point single-lens model. This supports the proposal by Lawrence et al. that microlensing is a plausible physical mechanism for extreme variability. However, we attribute the majority of our events to explosive stellar-related activity in the accretion disc: superluminous supernovae, tidal disruption events and mergers of stellar mass black holes.
\end{abstract}

Key words: methods: data analysis - techniques: photometric-surveys-quasars: general.

\section{INTRODUCTION}

Quasars are known to be a variable population, best described statistically via a damped random walk (DRW) model (e.g. Kelly, Bechtold \& Siemiginowska 2009, although see Kozlowski 2016a for a reappraisal). Their observed optical variability is typically a few tenths of a magnitude in amplitude with a characteristic time-scale of several months, but also showing larger variations over longer time-scales (MacLeod et al. 2012, 2016; Kozlowski 2016a). The variability amplitude and time-scale are anti-correlated with both luminosity and Eddington ratio, and correlated with black hole mass. The (extreme) tails of the variability distribution are less well characterized, however. While it is well established that blazars tend to exhibit large amplitude, short timescale variability, large amplitude variability on longer time-scales is not as well studied, and seems to probe a different population than blazars.

We have previously reported on a set of quasars showing periodic variability that is consistent with a population of supermassive black hole binaries with sub-parsec separation (D'Orazio, Haiman \& Schiminovich 2015; Graham et al. 2015a,b). Recently, a num-

^E-mail: mjg@caltech.edu ber of objects - so-called changing look quasars (LaMassa et al. 2015; MacLeod et al. 2016; Ruan et al. 2016) - have been reported, showing slow but consistent photometric variability $(\Delta m>1 \mathrm{mag})$ over several years coupled with spectral variability. Their optical spectra show emerging or disappearing broad emission line components, typically $\mathrm{H} \beta$. This is consistent with a change of type (Type 1-Type $1.2 / 1.5$ to Type $1.8 / 1.9-$ Type 2 or vice versa) and may be associated with a large change of obscuration or accretion rate. We have also reported a more extreme example of photometric and spectral variability exhibited by a BAL QSO, most probably experiencing a change in photoionization (Stern et al. 2017; see also Rafiee et al. 2016).

Clearly, a much larger sample of extreme variable quasars is needed to fully understand the different physical mechanisms that may be contributing to the observed phenomena and also to determine whether or how they relate to variability seen in the more general quasar population. Extreme quasars are, by definition, rare but the growing availability of large archives of astronomical time series, e.g. Sloan Digital Sky Survey (SDSS) Stripe 82 (Sesar et al. 2007), LINEAR (Sesar et al. 2011), Palomar Transient Factory (PTF; Rau et al. 2009) and Pan-STARRS (Kaiser et al. 2002), means that statistically valid samples of such objects can now be defined.

We have begun a systematic study of extreme quasar variability based primarily on the Catalina Real-time Transient Survey 
(CRTS ${ }^{1}$ Drake et al. 2009; Mahabal et al. 2011; Djorgovski et al. 2012). This is the largest open (publicly accessible) time domain survey currently operating, covering $\sim 33000 \mathrm{deg}^{2}$ in the range $-75^{\circ}<$ Dec. $<70^{\circ}$ (but avoiding regions within $\sim 10^{\circ}-15^{\circ}$ of the Galactic plane) to a depth of $V \sim 19-21.5$. Time series exists ${ }^{2}$ for approximately 500 million objects with an average of $\sim 300$ observations over an 11-year baseline.

In this work, we present a search for major flaring outbursts in active galactic nucleus (AGN). Subsequent papers will deal with other phenomena exhibited by extreme variable quasars (such as discussed above). Although there have been several reports of significant optical/UV outbursts in quiescent galaxies, consistent with superluminous supernovae or candidate tidal disruption events (TDEs) (Gezari et al. 2012; Chornock et al. 2014; Liu et al. 2014), those associated with active galaxies are much rarer as it can be difficult to distinguish a single significant event from more general variability. They have the potential, however, to inform about the structure and mechanics of the accretion disc and nuclear region.

Descriptions of major AGN flaring outbursts in the literature to date tend to deal with individual events. Meusinger et al. (2010) reported a significant UV flare in a quasar, Sharov 21, at $z=2.109$, seen through the disc of M31 (this had previously been misidentified as a nova event). The total outburst lasted $\sim 800 \mathrm{~d}$ with the source 3.3 mag brighter at maximum. The flare showed an asymmetric profile with a gradual increase followed by an abrupt rise and then a quasi-exponential decline $\left(t^{-5 / 3}\right)$ and a total bolometric energy release of $\sim 2 \times 10^{54} \mathrm{erg}$. It is consistent with a standard TDE scenario involving an $\sim 10 \mathrm{M}_{\odot}$ star and a $5 \times 10^{8} \mathrm{M}_{\odot}$ black hole (although microlensing is also considered as an alternate explanation). The TDE explanation, however, neglects the AGN nature of the host and the influence of the massive accretion disc and general relativistic effects on the dynamics of the stellar tidal debris.

Drake et al. (2011) discovered an extremely luminous optical transient within $150 \mathrm{pc}$ of the nucleus of a narrow-line Seyfert 1 galaxy, SDSS J102912+404220, at $z=0.147$. The total outburst lasted $\sim 400 \mathrm{~d}$ with the source $\sim 1.2 \mathrm{mag}$ brighter at maximum. It also showed an asymmetric profile with a slow increase and then a longer slow decline that was inconsistent with the expected $t^{-5 / 3}$ or $t^{-5 / 12}$ decline expected for TDEs. The proposed interpretation is an extremely luminous Type IIn supernova within the range of the narrow-line region of an AGN.

Lawrence et al. (2016) (hereafter L16) reported a search for large amplitude $(\Delta m>1.5 \mathrm{mag})$ nuclear changes in faint extragalactic objects in the PS1 $3 \pi$ survey compared against SDSS data over $11663 \mathrm{deg}^{2}$. Of the 76 transients detected, 43 are classed as 'slow blue hypervariable' AGN with smooth order of magnitude outbursts over several years, large colour changes between the SDSS and PS1 epochs, and weaker than average broad emission line strength in their spectra. A combination of changes in accretion state and large amplitude microlensing by stars in foreground galaxies seem to be the most likely explanations. Bruce et al. (2017) have also reported a more detailed analysis of four of the lensing candidates, with two well described by a simple single point-lens point-source model and the other two requiring a more complex lensing model. Although microlensing has the potential for mapping the inner structure of an AGN, these events only place minor constraints on the size of C III] and $\mathrm{Mg}$ II emission regions.
This paper is structured as follows. In Section 2, we present the selection technique for identifying major flaring activity and in Section 3, the data sets we have applied it to. We discuss our results in Section 4 and their interpretation in Section 5. We assume a standard Wilkinson Microwave Anisotropy Probe 9-yr cosmology $\left(\Omega_{\Lambda}=0.728, \Omega_{\mathrm{M}}=0.272, H_{0}=70.4 \mathrm{~km} \mathrm{~s}^{-1} \mathrm{Mpc}^{-1}\right.$; Jarosik et al. 2011) and our magnitudes are approximately on the Vega system.

\section{DATA SETS}

There are few data sets with sufficient sky and/or temporal coverage and sampling to support an extensive search for quasars exhibiting significant flaring. Most large studies of long-term quasar variability, e.g. SDSS with POSS (MacLeod et al. 2012) or Pan-STARRS1 (Morganson et al. 2014), consist of relatively few epochs of data spread over a roughly decadal baseline, which is sufficient to model ensemble behaviour but not to identify specific patterns in individual objects beyond a change of magnitude. CRTS represents the best data currently available with which to systematically define sets of quasars with particular temporal characteristics.

\subsection{Catalina Real-time Transient Survey}

CRTS leverages the Catalina Sky Survey (CSS) data streams from three telescopes - the $0.7 \mathrm{~m}$ Catalina Sky Survey Schmidt, $1.5 \mathrm{~m}$ Mount Lemmon Survey telescopes in Arizona and the $0.5 \mathrm{~m}$ Siding Springs Survey Schmidt in Australia - used in a search for Near-Earth Objects, operated by Lunar and Planetary Laboratory at University of Arizona. CRTS covers up to $\sim 2500 \mathrm{deg}^{2}$ per night, with four exposures per visit, separated by $10 \mathrm{~min}$, over 21 nights per lunation. New cameras in Fall 2016 with larger fields-of-view will increase the nightly sky coverage. All data are automatically processed in real time, and optical transients are immediately distributed using a variety of electronic mechanisms. ${ }^{3}$ The data are unfiltered but are broadly calibrated to Johnson $V$ from 2MASS data (see Drake et al. 2013 for details). The accuracy of the $V$-band photometry is highly dependent on source colour but comparison with Landolt standard stars has shown that the colour correction is small for blue objects. The effect on quasar variability should therefore be minimal. The full CRTS data set ${ }^{4}$ contains time series for approximately 500 million sources.

We note that the published error model for CRTS is incorrect. The photometric uncertainties were originally determined ${ }^{5}$ via an empirical relationship between source flux and the observed photometric scatter. This relation was derived from 100000 isotropically selected sources that exhibited no significant sign of variability based on their Welch-Stetson variability index. However, errors at the brighter magnitudes are overestimated and those at fainter magnitudes ( $>18$ ) are underestimated (Palaversa et al. 2013; Drake et al. 2014; Vaughan et al. 2016). We have derived a multiplicative correction factor from CRTS observations of 350000 sources in the Stripe 82 Standard Star catalogue (Ivezic et al. 2007) that ensures that the mode of the reduced chi-squared variability in magnitude bins of width of $\Delta$ mag $=0.05$ is centred at unity (see Fig. 1).

We have extracted a number of samples from CRTS in which to search for significant AGN flaring and these are summarized in

\footnotetext{
${ }^{1} \mathrm{http}: / /$ crts.caltech.edu

${ }^{2} \mathrm{http}: / / \mathrm{www} . c a t a l i n a d a t a . d a t a$
} 


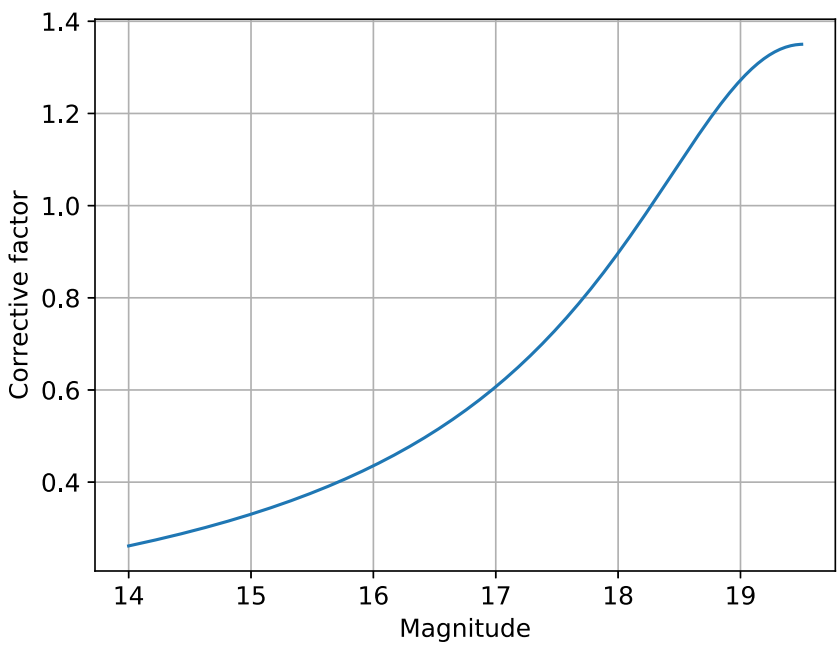

Figure 1. Corrective factor for photometric errors in CSS.

Table 1. Repeat instances of sources between different samples are ignored, i.e. they are included in the first sample but not subsequent ones. The total number of unique sources employed is 2127266 and their sky coverage and $V$ magnitude distribution are shown in Figs 2 and 3, respectively. We also note that none of these sources has fewer than 10 observations in their light curve. We apply the same preprocessing steps described in Graham et al. (2015b) to all light curves.

\subsubsection{Spectroscopically confirmed quasars}

The Million Quasars (MQ) catalogue ${ }^{6}$ v3.7 contains all spectroscopically confirmed type 1 QSOs (309 525), AGN (21 728) and BL Lacs (1573) in the literature up to 2013 November 26 and formed the basis for the results of Graham et al. (2015b). We have extended this with an additional 297301 spectroscopically identified quasars in the SDSS Data Release 12 (Paris et al. 2016). We cross matched this combined quasar list against the CRTS data set with a 3 arcsec matching radius and find that 334402 confirmed quasars are detected by CRTS. Of these, 12867 do not have enough observations $(n<5)$ for any peak to be identified, leaving a data set of 321535 quasars.

\subsubsection{The Extreme Deconvolution Quasar Sample}

DiPompeo et al. (2015) have combined (forced) WISE W1 and $W 2$ photometry with SDSS colours using extreme deconvolution to produce a probabilistic catalogue of 5537436 quasar candidates in SDSS DR8. Of these, 1730760 have a corresponding match in CRTS. We select those candidates with $p_{\text {QSO }}>0.99999$, which gives 589350 sources, of which 425767 are not previously known quasars and have $n>5$. We note that of the 264821 confirmed (from MQ) quasars in Extreme Deconvolution Quasar Sample, 128104 (48.4 per cent) have $p_{\mathrm{QSO}}>0.99999$ and 15 per cent have $p_{\mathrm{QSO}}<$ 0.95 . Each quasar candidate in the catalogue has also been assigned a photometric redshift which we use when required for cosmological calculations.

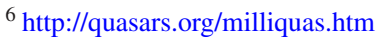

\subsubsection{WISE-selected AGN}

As part of defining a joint variability and colour-selected quasar catalogue from CRTS data (Graham et al., in preparation), we have identified 233373 WISE AGN on the ecliptic. These have W $<15$ and $\mathrm{W} 1-\mathrm{W} 2>0.8,|b|>10$ and $-15 \leq \delta \leq 15$ (Stern et al. 2012; Assef et al. 2013). They also do not appear in the known quasar or XDQSO samples (although note that with these selection criteria there are 35731 duplicates with MQ and 109186 duplicates with XDQSO).

\subsubsection{Transients}

To date, CRTS has detected 13149 optical transients (see http://crts.caltech.edu for details). Many of these are not associated with any previously detected source, indicating that these sources were below the survey detection limit in their (more) quiescent state. 3628 of these show an apparent aperiodic variability that shows increasing amplitude on long time-scales whilst lacking any obvious short time-scale outbursts or rapid variations. This variability is consistent with being an AGN and ancillary data, such as colour, spectra, coincidence with a radio source, etc., are used when available to support the identification. None of these sources has been previously classified as an AGN. Given the nature of their initial detection, we have also included these in our candidate list.

\subsubsection{Variables}

CRTS is the basis for many studies of variability in astronomical populations and in an initial characterization of general source variability in CRTS, we identified a set of 1 million objects with Stetson $J / K$ values above magnitude-dependent fiducial values in the local field (see Drake et al. 2014 for more details). Although these sources remain largely unclassified, we are including them in this analysis since they can be used to estimate flaring statistics for a general variable population in addition to providing more AGN candidates. We have determined QSO variability statistics and WISE colours for these and select 27003 candidates that are not part of any of the other data sets described here.

\section{IDENTIFYING MAJOR FLARING ACTIVITY}

L16 define an AGN as a slow blue hypervariable if it has brightened by an order of magnitude $(|\Delta g|>1.5)$ over the course of a decade in a smooth fashion and is now mostly fading (but may also still be increasing in flux). The strength of its $\mathrm{Mg}$ II emission may be weaker as well than what would be expected, given its luminosity. We are interested in finding any AGN source with outburst activity that can be characterized as statistically significant. This relates not only to the strength of the activity but also to its duration and morphology. Because of the novelty of this field, the relevant distributions are a combination of the theoretical and the phenomenological.

\subsection{The amplitude of variability}

Although a number of examples of extreme quasar variability are known, particularly in the blazar population, to date the statistics of extreme variability have only been marginally constrained. Assuming a CAR(1)/DRW model for quasar variability in a sample of 33881 quasars with at least two epochs of SDSS or POSS photometry, MacLeod et al. (2012) found that the distribution of magnitude difference for a given time lag is exponential. This is a cumulative 
Table 1. List of CRTS samples searched for significant AGN flaring activity. The number of unique sources refers to objects only appearing in that data set - repeat instances in other data sets are ignored; e.g. a source identified both as a known spectroscopic quasar and by WISE will appear under 'Known QSOs' only. With the variables data set, the asterisked number is the total number of sources examined but the number in parentheses is the number of objects with AGN-consistent variability and WISE colours.

\begin{tabular}{lccc}
\hline Sample & $\begin{array}{l}\text { Number of unique } \\
\text { sources }\end{array}$ & $\begin{array}{c}\text { Number of AGN } \\
\text { flare candidates }\end{array}$ & $\begin{array}{c}\text { Number of candidates excluded } \\
\text { due to more than one significant flare }\end{array}$ \\
\hline Known QSOs (A) & 321535 & 13 & 65 \\
XDQSO (B) & 324338 & 26 & 32 \\
WISE (C) & 233373 & 10 & 27 \\
Transients (D) & 2965 & - & - \\
Variables (E) & $1290055^{*}$ & 2 & 4 \\
& $(27003)$ & 51 & 128 \\
Total & $2127266^{*}$ & & \\
\hline
\end{tabular}

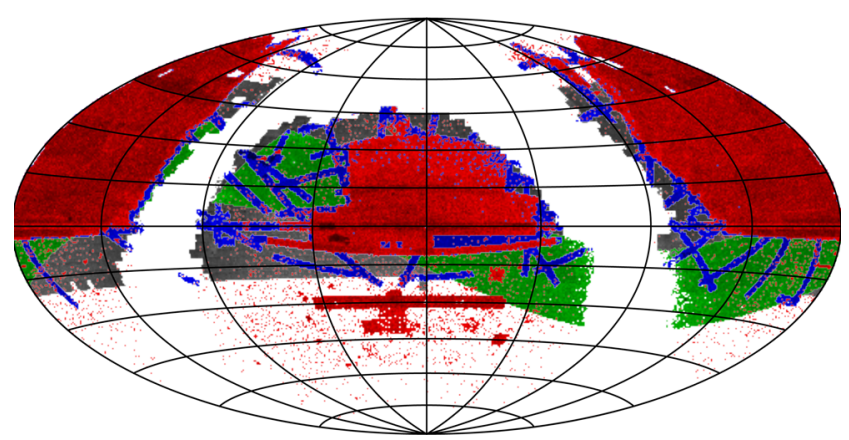

Figure 2. Sky coverage (RA, Dec., Mollweide projection) for sources considered in this paper. The colours represent the subsamples: known QSOs (red), XDQSO (blue), WISE (green) and variables (grey).

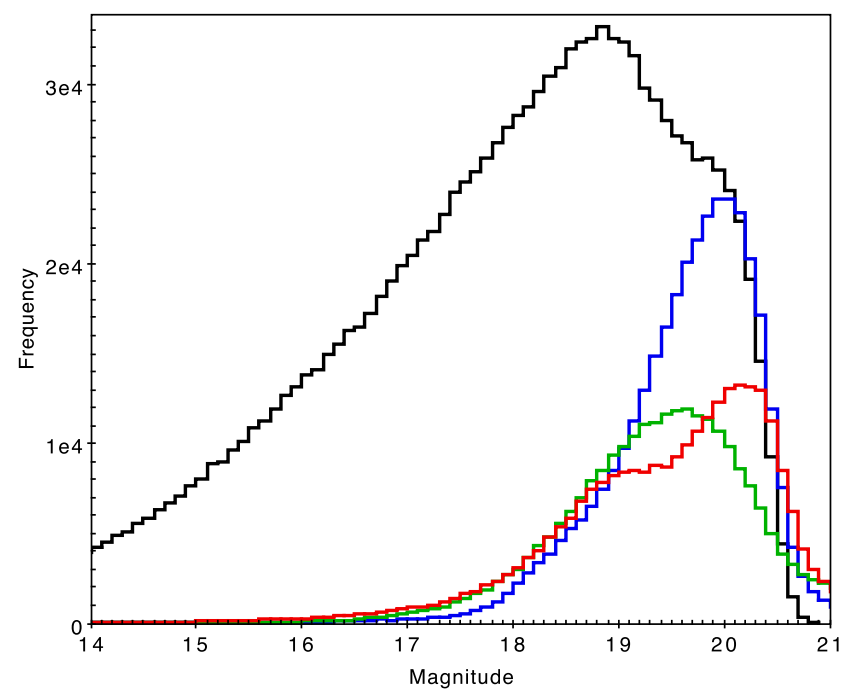

Figure 3. Relative $V$ magnitude distributions for sources considered in this paper. The colours represent known QSOs (red), XDQSO (blue), WISE (green) and variables (black).

effect of averaging over a range of different characteristic timescales, $\tau$, and variability amplitudes, $S F_{\infty}$. From a joint SDSS-PS1 analysis, L16 claim that somewhere between 1 in 1000 and 1 in 10000 AGNs show extreme variability $(|\Delta g|>1.5)$ over the period of a decade.

The sampling and time coverage of CRTS data allow us to validate these claims. For each quasar in the known data set (A), we have calculated the median absolute magnitude difference as a func-

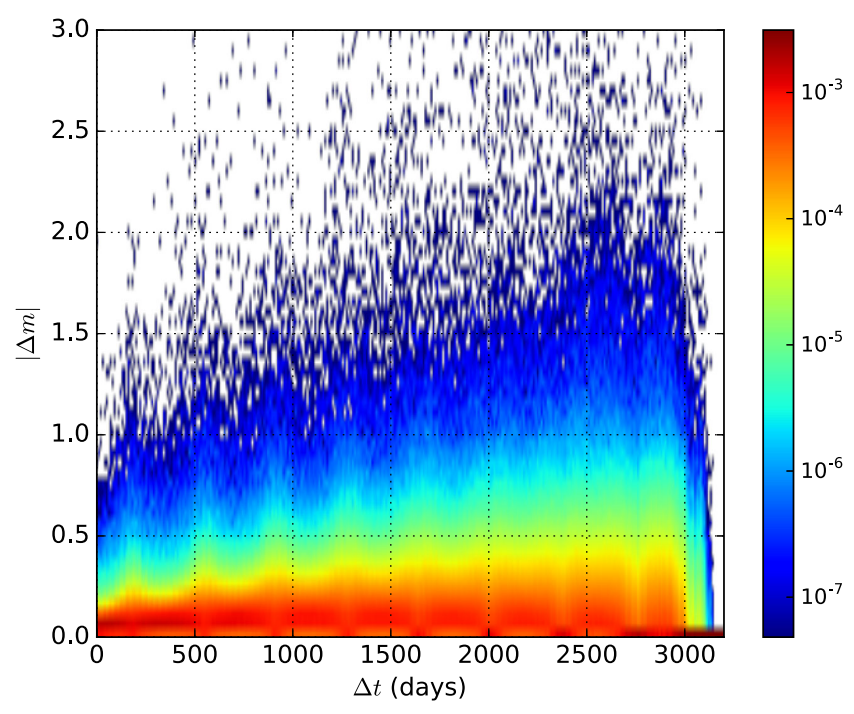

Figure 4. Joint probability distribution of magnitude difference and time lag from the ensemble of known quasars. The sampling effects of annual cycles can be clearly seen.

tion of time lag, $\operatorname{med}(|\Delta m(t)|)$, spanning a range of 10-3200 d in bins of $\mathrm{d} t=10 \mathrm{~d}$. Fig. 4 shows the ensemble joint probability distribution, from which we can determine the expected number of quasars with variability $|\Delta m|>\Delta m_{0}$ over a time-scale $t \geq t_{0}$. Fig. 5 shows the observed cumulative magnitude differences against the predicted behaviour for three different time lags. Previous structure function-based analyses (de Vries et al. 2005; MacLeod et al. 2012; Morganson et al. 2014; Kozlowski 2016a) have shown that the amplitude of AGN variability increases with longer time-scales and the behaviour we see in our data set is consistent with this.

Fig. 6 shows the marginal probability distribution for $|\Delta m|$. This suggests that extreme variability is an order of magnitude rarer than previous claims and that a limit of $|\Delta m|>1.0$ of $\sim 10 \mathrm{yr} p(|\Delta m|>$ 1) $\sim 10^{-3}$ is sufficient to define significant flaring activity.

\subsection{Selection criteria}

We model the median activity of a source over time with a linear fit to its light curve. We derive the slope and best offset using the Thiel-Sen estimator (the median slope between all pairs of points). Candidate flares are identified as contiguous sets of points brighter than the median level and each is characterized in terms of its temporal span (tspan), median (and peak) amplitude (amp) 


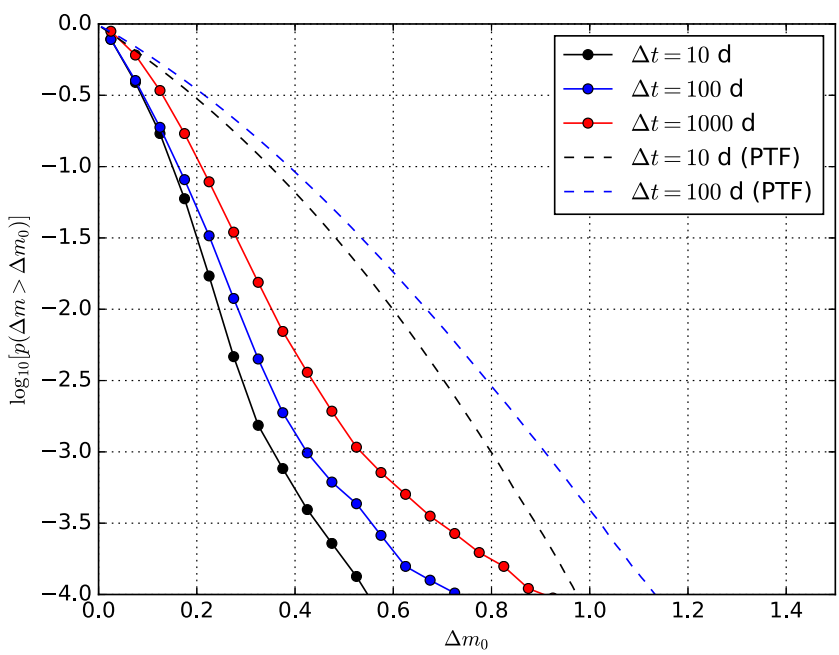

Figure 5. Cumulative absolute magnitude difference seen in CRTS data from different time lags compared to $r$-band predicted values for PTF for $r$ $<20.6$ from a DRW model of quasar variability (MacLeod et al. 2012). For the purposes of this analysis, CRTS and PTF are comparable - an $r<20.6$ limit is equivalent to $V_{\text {CRTS }}<20$.

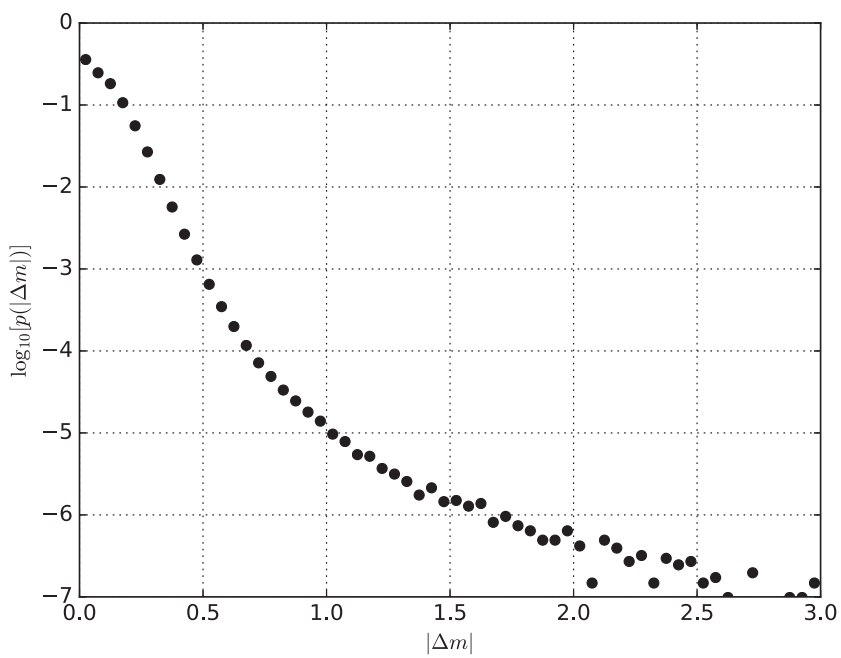

Figure 6. Marginal probability distribution for median absolute magnitude differences $|\Delta m|$ over 3,200 d.

above the median level (to help identify false positives resulting from poor-quality photometry), and number of contributing points (npts). Since we are interested in the joint distribution of these three features, we found that we could also represent this through a single parameter, defined as the logarithm of the geometric mean of the three features:

pksig $=\frac{1}{3}\left[\log _{10}\left(a m p_{\text {norm }}\right)+\log _{10}(\right.$ npts $)+\log _{10}($ tspan $\left.)\right]$.

Note that $a m p_{\text {norm }}$ is the normalized median amplitude (see below). A typical given source will contain several such flares and we use the median absolute deviation (MAD) of its flare amplitudes to define a baseline level of flare activity for the source against which to identify significant flares.

The scale of variability is a function of magnitude: fainter objects show larger MAD as there is a larger noise contribution for low signal-to-noise ratio (faint) sources (see Fig. 7). Using CRTS data for 72634 standard stars in the Stripe 82 region (Ivezic et al.

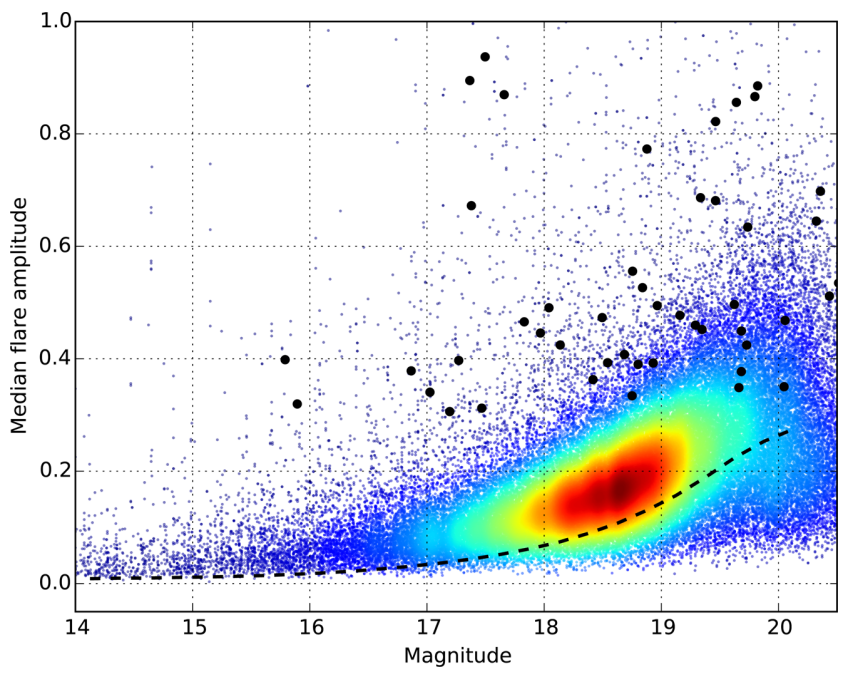

Figure 7. Median flare amplitude as a function of magnitude for 87017 spectroscopically confirmed known quasars with some degree of flaring (time span $>100 \mathrm{~d}$ and more than 10 observations in the flare). The black dashed line indicates the MAD as a function of magnitude for 72634 standard stars in Stripe 82, which is used to normalize the variability scale.

2007) with a magnitude range of $V=14-20.5$, we have derived a normalization based on the median MAD value for a given magnitude to ensure that objects with equivalent variability strength (irrespective of magnitude) can be compared.

We also characterize any flares in terms of shape parameters using the (translated) Weibull distribution. This has been shown to be a convenient function for empirically fitting the shapes of flares (Huenemoerder et al. 2010). The probability distribution is:

$f(p ; a, s)=\left(\frac{a}{s}\right) p^{a-1} \mathrm{e}^{-p^{a}}$

$p=\left(t-t_{0}\right) / s$

in which $a$ is a shape parameter ( $a>0$ ), the scale (or width) is specified by $s(s>0)$, and the offset (location) is given by $t_{0}\left(t_{0} \geq 0\right)$; the independent coordinate is $t\left(t \geq t_{0}\right)$. For fixed $a$, increasing values of $s$ stretch out/broaden the function and for fixed $s$, increasing values of $a$ sharpen the peak (see Fig. 8). We fit $A f(p ; a, s)+R_{0}$ (relative to the linear fit representing the median source activity), where $A$ is an amplitude and $R_{0}$ is the baseline magnitude. Note that this is just a location-scale transformed version of the Weibull distribution.

Given the apparent rarity of significant flares, we expect such objects to be population outliers in the parameter space defined by these characterizing features. From the two examples in the literature (Meusinger et al. 2010; Drake et al. 2011), we might expect the outburst to last at least $300 \mathrm{~d}$ (in the observed frame) and have a peak of at least 1 mag above the normal level of activity that is consistent with our predictions in the last section. We note that non-Type IIn supernovae have typical decay times of between 20 and $150 \mathrm{~d}$ and so most of these phenomena would be excluded. We use a lower cutoff of 30 observations in the light curve to ensure that the flares are sufficiently well-sampled.

We expect to see only one significant flare over the time covered by the light curve and exclude those with more - these are typically cataclysmic variables or carbon stars, a consequence of including quasar candidates in our sample. We also want to exclude blazars as we are specifically interested in individual flaring events from a (semi-)quiescent state rather than general continuous large 

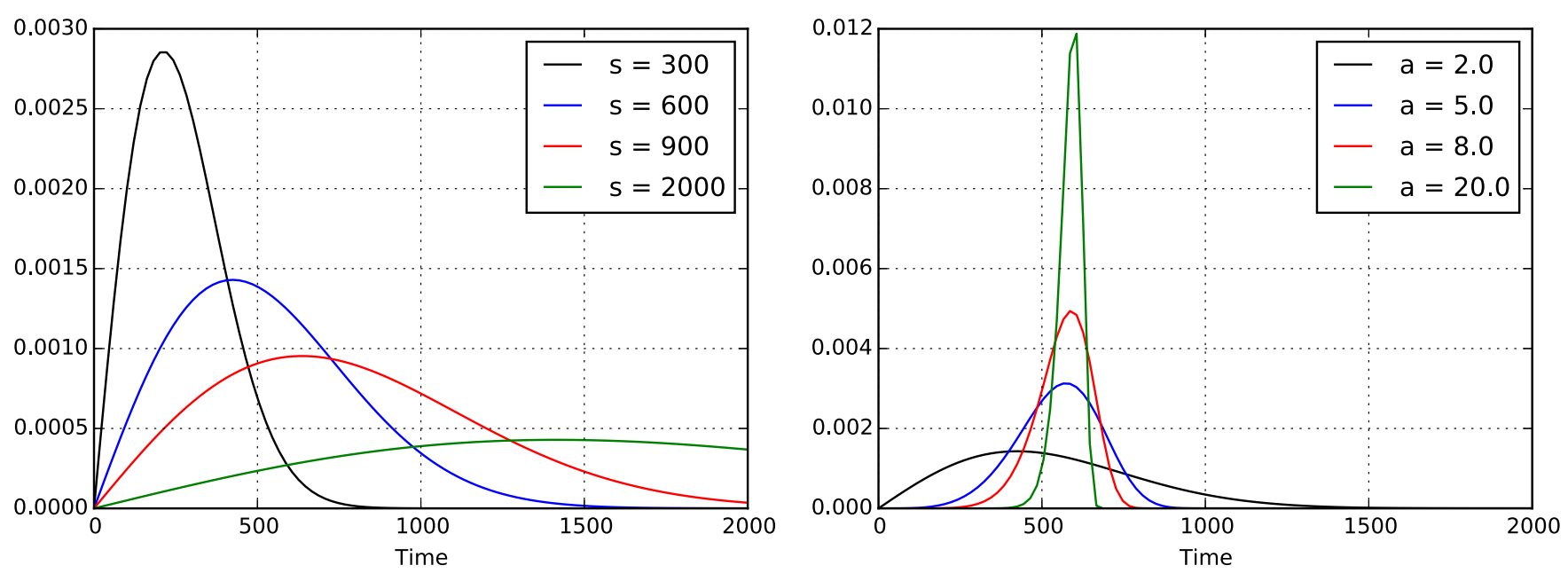

Figure 8. Sample Weibull distributions. The left plot shows varying the scale for fixed shape $(a=2)$ and the right changing the shape for fixed scale $(s=600)$.

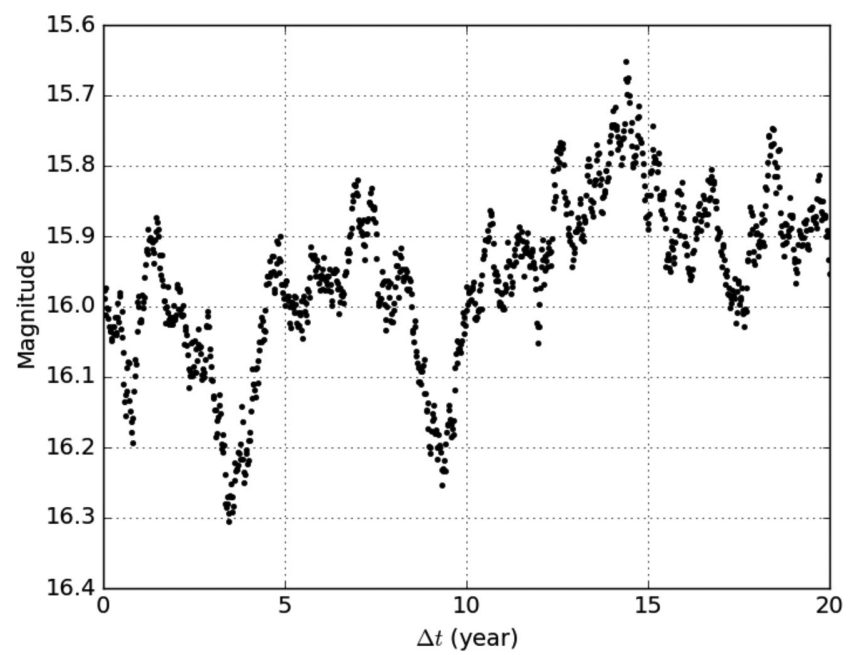

Figure 9. A mock light curve for a DRW process.

amplitude (>1 mag) flaring activity. One possible source of contamination is stray light (e.g. diffraction spikes, reflections) from a nearby bright source, low surface brightness galaxy or genuine blend. We applied the same criteria as described in Graham et al. (2015b) to identify such sources and exclude them from further consideration.

\subsection{Mock data}

Autoregressive processes, such as those used to describe quasar variability (Kelly et al. 2014), have correlated (red) noise [characterized by a power spectrum of the form $P(f) \propto v^{-2}$ ] which can introduce features in their time series, such as dips and humps (see Fig. 9). To ensure that the features we are identifying are not just noise artefacts (note that these are intrinsic to the source and not associated with any form of measurement noise), we can determine the expected distribution of noise-related features in terms of the characterizing measures we are employing to describe the real flares - median amplitude and time span - from simulated time series generated by a particular stochastic model. As in previous analyses (Graham et al. 2014, 2015b), we simulate quasar variability via a DRW pro-
Table 2. The magnitude-binned means and covariances of the DRW parameter distributions.

\begin{tabular}{lcccccc}
\hline Magnitude & $\langle\tau\rangle$ & $\left\langle\sigma^{2}\right\rangle$ & $C_{00}$ & $C_{01}$ & $C_{10}$ & $C_{11}$ \\
\hline 14.25 & 1.966 & -2.422 & 1.142 & 0.173 & 0.173 & 0.379 \\
14.75 & 2.186 & -2.531 & 0.772 & 0.105 & 0.105 & 0.476 \\
15.25 & 2.160 & -2.494 & 1.046 & 0.343 & 0.343 & 0.469 \\
15.75 & 2.375 & -2.314 & 0.768 & 0.208 & 0.208 & 0.407 \\
16.25 & 2.535 & -2.339 & 0.636 & 0.197 & 0.197 & 0.312 \\
16.75 & 2.613 & -2.225 & 0.561 & 0.191 & 0.191 & 0.266 \\
17.25 & 2.753 & -2.137 & 0.299 & 0.116 & 0.116 & 0.218 \\
17.75 & 2.798 & -2.047 & 0.252 & 0.120 & 0.120 & 0.201 \\
18.25 & 2.794 & -2.018 & 0.228 & 0.106 & 0.106 & 0.172 \\
18.75 & 2.704 & -1.938 & 0.266 & 0.108 & 0.108 & 0.181 \\
19.25 & 2.580 & -1.758 & 0.306 & 0.098 & 0.098 & 0.160 \\
19.75 & 2.409 & -1.611 & 0.406 & 0.074 & 0.074 & 0.115 \\
20.25 & 2.221 & -1.602 & 0.507 & 0.033 & 0.033 & 0.080 \\
\hline
\end{tabular}

cess characterized by a time-scale $\tau$ and an amplitude $\sigma^{2}$. A (zero centred) data point $m_{i+1}$ at time $t_{i+1}$ is given by

$m_{i+1}=m_{i} \mathrm{e}^{-\Delta t / \tau}+G\left[\sigma^{2}\left(1-e^{-2 \Delta t / \tau}\right)\right]$,

where $G\left(s^{2}\right)$ is a Gaussian deviate with variance $s^{2}$ and $\Delta t=t_{i+1}$ $-t_{i}$. A second Gaussian deviate is added to each value to represent observational noise: $y_{i}=m_{i}+G\left(n_{i}^{2}\right)$, where $n_{i}$ is the error at time $t_{i}$ - this ensures heteroscedastic errors as in the real light curves. Previously, we have drawn $\tau$ and $\sigma^{2}$ from the rest-frame fitting functions determined by MacLeod et al. (2010) but we have now calculated $\left(\tau, \sigma^{2}\right)$ for all known quasars via Gaussian process regression. We evaluated the mean and covariance of these in $\Delta m=$ 0.5 mag bins and now draw a magnitude-dependent $\operatorname{random}\left(\tau, \sigma^{2}\right)$ from the joint Gaussian with the appropriate mean and covariance (see Table 2).

\section{RESULTS}

We applied our flare identification algorithm to 2127266 CRTS light curves and found 91321768 candidate flares (see Fig. 10). An initial selection is provided by selecting those flares with a time span longer than $300 \mathrm{~d}$, a normalized median amplitude greater than a factor of 2.5 and sampled by 30 or more observations. This produces 19150 flares from 14592 distinct sources (known AGN 

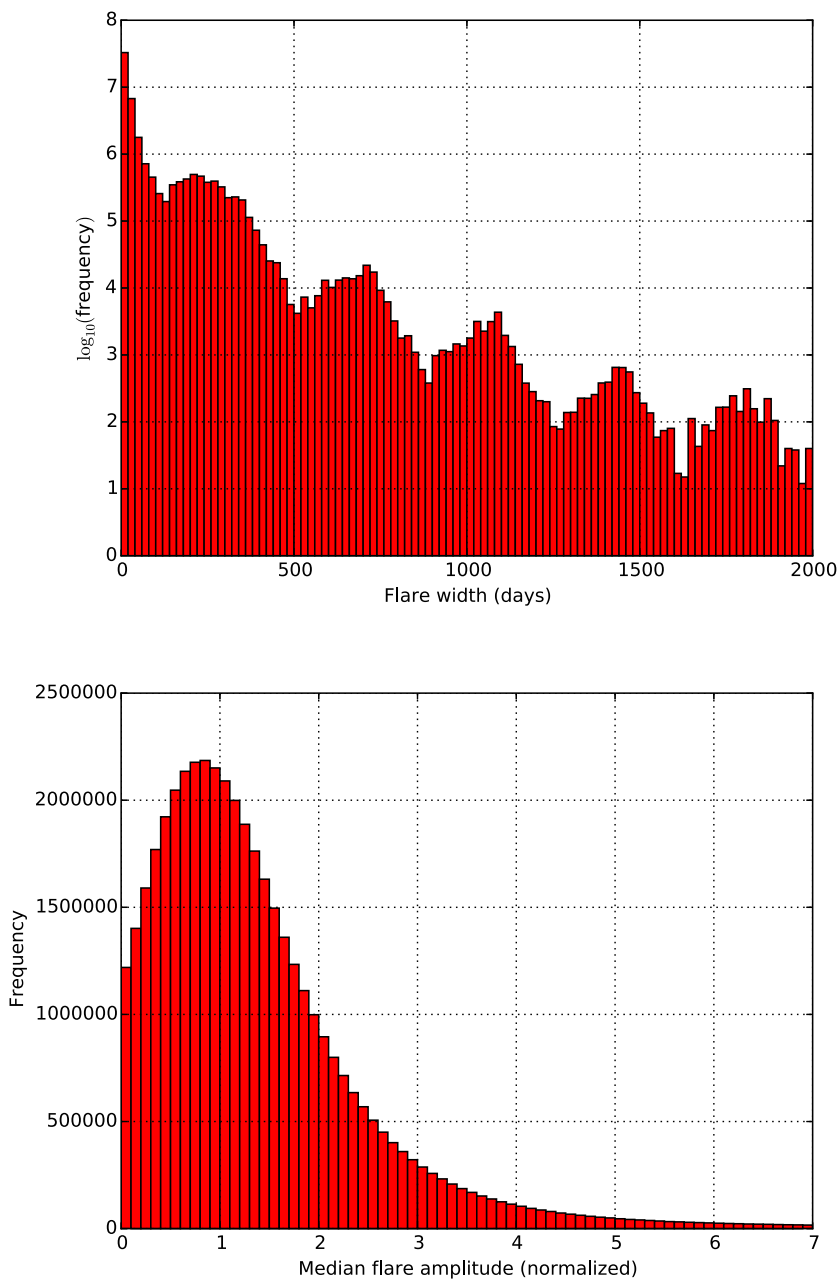

Figure 10. Frequency distribution for flare time spans and median amplitudes. The apparent periodicity in the flare time span distribution is due to the sampling effects of annual cycles as in Fig. 4.

or AGN candidates). Removing those associated with nearby bright stars or blended sources reduces this to 13527 . We have also ignored activity from 529 known blazars (using the class designation in MQ and the BZCAT v5.0 catalogue of blazars (Massaro et al. 2015)). For comparison, we find 1602 from a simulated realization of the known quasars sample containing 321535 sources and the same selection criteria. This suggests that the number of real flares is more than expected.

We are interested in those sources where the flaring represents a noticeable change from a lower or quiescent state over the time span of the light curve, i.e. where the flaring activity is significant relative to the baseline activity of the source. From the distribution of peak amplitude against the significance of the flare:

sig $=\frac{p k s i g-p k s i g_{\mathrm{med}}}{\operatorname{mad}(p k s i g)}$;

we identify a set of 585 candidates (see Fig. 11) with peak amplitude $>0.5 \mathrm{mag}$ ( 154 sources from the simulated data pass this criterion). This is more inclusive than the $\Delta m=1$ limit we argue for in Section 3.2, but captures the significant large amplitude outliers from the sample distribution. We also note in Table 1 the number of candidates that were excluded due to the presence of more than one significant flare in the light curve.

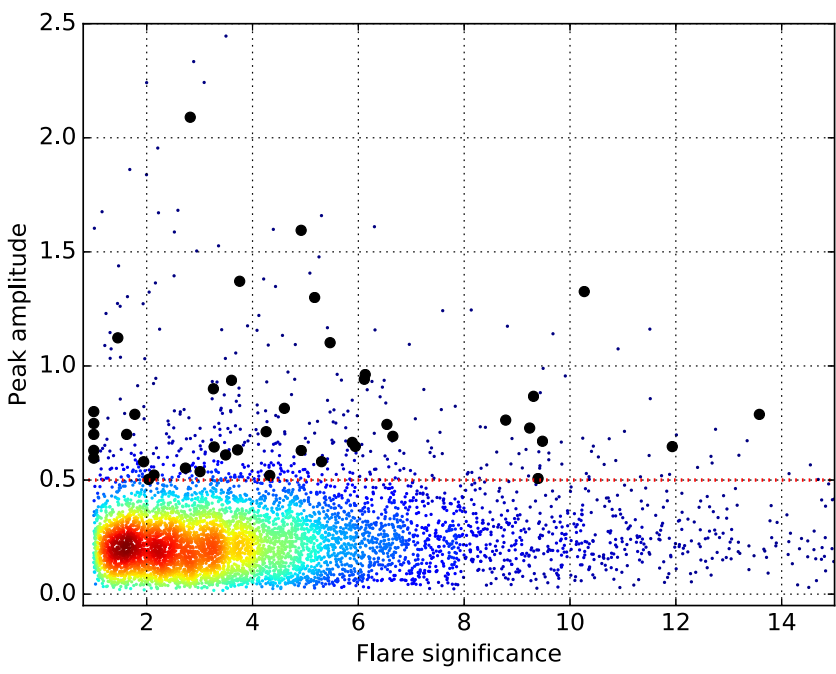

Figure 11. Distribution of peak flare amplitude against the flare significance. The small points are colour coded according to the local density of points. The dotted red line indicates the 0.5 cutoff value we use and the larger black dots are the identified flare candidates.

The flaring could still be the result of correlated noise (regular quasar variation) rather than a specific physical mechanism. For each candidate, we therefore construct a comparison time series with the primary flare removed. We describe both time series (with and without the flare) as a DRW process ${ }^{7}$ via Gaussian process regression and incorporating heteroscedastic errors (using the PYTHON code $\mathrm{GPy}^{8}$ ). These models are parametrized by a characteristic time-scale $\tau$ and a variance $\sigma^{2}$. If the flaring activity is consistent with the general variability of the quasar (arising from correlated noise in a DRW) then the sets of $\left(\tau, \sigma^{2}\right)$ values for the two light curves should agree within the confidence limits on the parameters. However, differing values indicate that the flaring is incompatible with such a model.

Fig. 12 shows the distribution of the parameter differences between the two time series. Most of the sources have compatible DRW descriptions with or without the flare (clustered around the origin); however, a subset of 48 does not and we consider these to be major flare candidates. We also note that there are three objects where there is no discernible lower state, i.e. the 'flare' represents the whole light curve. Determining a parameter difference is therefore not possible with these but the parameter values from describing these with a DRW are sufficiently different from the general population that we regard them as a separate set of 'superflare' candidates [although changing-look candidates are also on the top right of the DRW parameter $\tau-\sigma^{2}$ distribution (Graham et al., in preparation)].

Table 6 lists all the flare candidates and their light curves are shown in Fig. 13. Where possible, we have obtained spectroscopic redshifts for candidates without existing spectra using the Palomar 200 arcsec and Keck telescopes (see the Appendix for more details). Photometric redshifts are used for those remaining candidates without spectra (which are typically outside the SDSS footprint). These are taken from the XDQSO catalogue or in those cases where one is not available, calculated using the XDQSO IDL code ${ }^{9}$ with SDSS

\footnotetext{
${ }^{7}$ This is a purely statistical description of the variability and makes no inferences about the physical processes contributing to variability.

${ }^{8} \mathrm{http}: / /$ gitlab.com/GPy

${ }^{9} \mathrm{http}: / /$ xdqso.readthedocs.org/
} 


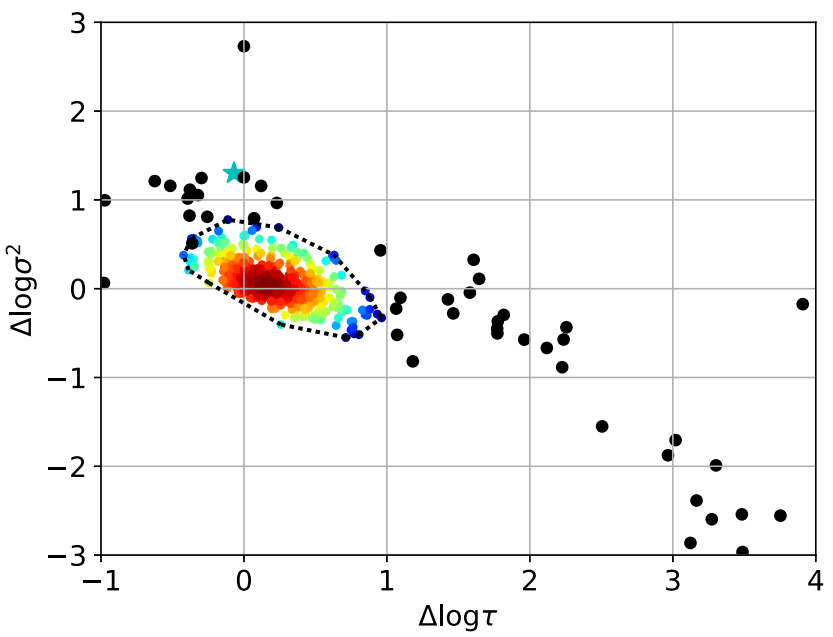

Figure 12. Distribution of DRW parameter differences between the time series with and without the primary flare. The black dotted line indicates the contour level used to identify outliers (flare candidates). Two outliers lie outside the bounds of the plot. The blue star denotes the position of Sharov 21 with this analysis.

magnitudes and forced WISE photometry from Lang, Hogg \& Schlegel (2016). We fit each flare candidate with a Weibull distribution (as described in Section 3.2) to allow further characterization of the phenomena that we are detecting and the parameters are reported in the table.

We also estimate the total energy output by each flare. The absolute magnitude of a source is given by:

$M_{V}=m_{V}-A_{V}-D M-K_{V}$,

where $A_{V}$ is the extinction, $D M$ is the distance modulus and $K_{V}$ is the $K$-correction. We obtain ${ }^{10}$ extinction values at the source position from the Schlafly \& Finkbeiner (2011) recalibration of the Schlegel et al. (1998) reddening maps. We assume a $K$-correction of: $K=$ $-2.5(\alpha+1) \log (1+z)$ for a power-law SED of $F_{v} \propto v^{\alpha}$ with $\alpha=$ -0.5 . The bolometric luminosity in band $X$ can be defined as:

$L_{\mathrm{bol}, X}=b_{X} \mathrm{~L}_{\odot, X} 10^{\left(\mathrm{M} \odot_{, X}-M_{X}\right) / 2.5}$

where the solar constants for filter $V$ are $\mathrm{M}_{\odot, V}=4.83$ and $\mathrm{L}_{\odot, V}$ $=4.64 \times 10^{32} \mathrm{erg} \mathrm{s}^{-1}$ and $b_{X}$ is the bolometric correction. We report the total integrated bolometric luminosity without bolometric correction for each source in Table 6 (see also Fig. 14). As a check, Drake et al. (2011) give a value of $8.5 \times 10^{50} \mathrm{erg}$ for this quantity for the source J102913+404420, which compares well with our estimate of $8.63 \times 10^{50} \mathrm{erg}$. They also determine a mean bolometric correction of $\bar{b}_{V} \sim 15$ giving an integrated bolometric luminosity of $\sim 1.3 \times 10^{52} \mathrm{erg}$. Although the quantity can be a source- and time-dependent quantity, particularly during the flaring activity, we will adopt a canonical value of $b_{V}=10$ for the candidates here.

\section{DISCUSSION}

In this section, we consider possible physical explanations for the extreme AGN variability we have detected.

\footnotetext{
${ }^{10}$ http://irsa.ipac.caltech.edu/applications/DUST/
}

\subsection{Microlensing}

From 3 yr of PS1 data, L16 identified 49 AGNs, most previously unknown, with variability ostensibly similar to what we have reported: smooth order of magnitude outbursts over several years. They also identified an additional 15 AGNs in SDSS Stripe 82 data with $\Delta g>$ 1.5 over a decadal baseline. They propose that microlensing by the close passage of a single star in an intervening galaxy, or a caustic caused by a small number of stars, is the most likely explanation for such rare temporary large amplitude events. Furthermore, a canonical model with a source at $z=1$ and a $1 \mathrm{M}_{\odot}$ mass star in motion within a galaxy at $z=0.25$ with a transverse velocity of $300 \mathrm{~km} \mathrm{~s}^{-1}$ predict a characteristic time-scale and event rate within an order of magnitude of that seen. Sharov 21 (Meusinger et al. 2010) is considered a canonical example in the existing literature, although it is slightly better fit by a two-star lens model.

Assuming that both the source and the lens are pointlike and that their relative motion is linear, the magnification associated with microlensing can be approximated as:

$\mu(t)=\frac{F_{v, \mathrm{obs}}(t)}{F_{v, g s}}=\frac{u(t)^{2}+2}{u(t) \sqrt{u(t)^{2}+4}}$,

where $u(t)$ is the angular distance between the source and the lens in units of the Einstein angle, and $F_{v, o b s}(t)$ and $F_{v, g s}$ are the observed flux density at time $t$ and the mean flux density in the ground state, respectively. This produces a symmetric profile and we can use the Weibull characterization of each flare as an indicator of how likely this model seems.

We can test whether the range of Weibull parameters that we find is consistent with that expected from a microlensed population of quasars observed with a CRTS-like survey. For a given source at redshift $z_{\mathrm{s}}$, we assume a lens at half the corresponding angular diameter distance with a transverse velocity of $300 \mathrm{~km} \mathrm{~s}^{-1}$ and mass drawn from the galactic stellar mass function of Chabrier (2003). We also assume a minimum impact parameter, $u_{\text {min }}$, drawn from a uniform distribution in the range $0.063-0.63$, where the lower bound comes from the maximum amplitude detected $(\Delta m=3)$ and the upper bound from the minimum amplitude required $(\Delta m=0.5)$ to be detected by our process. We model the redshift distribution of the quasar population from that of the CRTS + XDQSO data with mag $<19$ to allow a reasonable detection of a flare (note that the mean magnitude of the flare candidates is 19.05.). We also model the time difference between successive observations of CRTS light curves to generate equivalent irregular sampling patterns over the timescale of a lensing event. We use a ground state magnitude drawn from the magnitude distribution of the same CRTS + XDQSO data set used for the redshifts. Finally, we add heteroscedastic Gaussian noise terms to all magnitudes drawn from a Gaussian with mean and standard deviation equal to that of typical measurement errors in CRTS data as a function of magnitude.

With these priors (summarized in Table 3), we generated 100000 simulated single-lens events and fit Weibull models to the resulting light curves using the same procedure as before. Fig. 15 shows the distributions of the Weibull shape $(a)$ and scale $(s)$ parameters from the simulated flares as well as those from our flare candidates. It is interesting to see whether there is any relationship between the duration of a flare and its symmetry. Fig. 16 shows the distribution of the skewness of the Weibull fit for each flare (see Appendix B for a derivation) versus the duration of the flare. It is clear that the symmetry of the flare is largely independent of its duration. We have also tested simple two-lens models to add a degree of asymmetry to the simulated flare: each component is treated as a single star with 

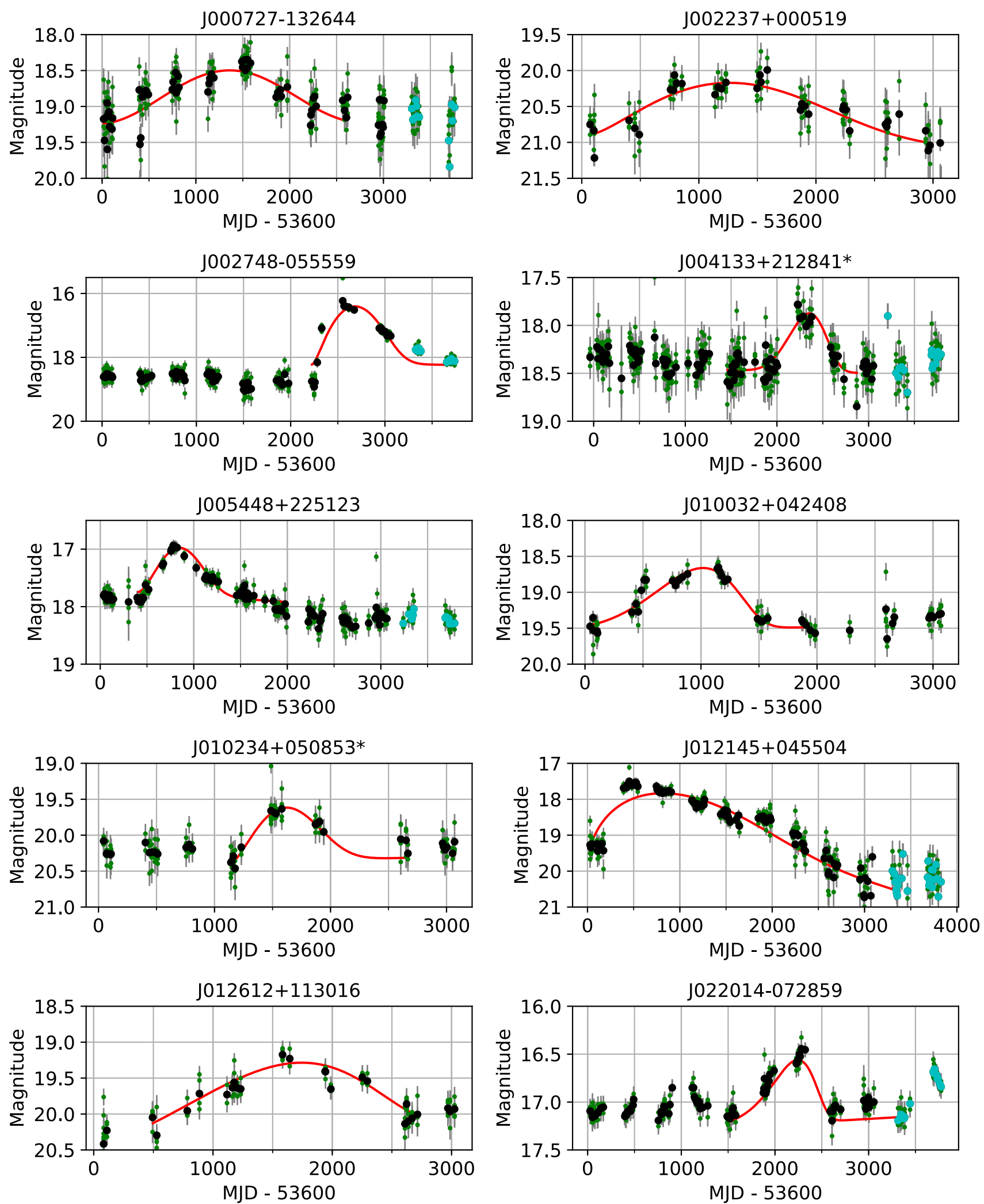

Figure 13. Light curves for flaring candidates. CRTS data (DR2: black, post-DR2: cyan) are shown; complementary data from the LINEAR (blue) (Sesar et al. 2011) and PTF (red) (Rau et al. 2009) surveys are included where available. The light curve for Sharov 21 is also shown for comparison. The line in each plot shows the best-fitting Weibull distribution to the identified flare. Note that this is relative to a linear model for the median activity of the source. Sources with a corresponding lens model in Fig. 17 are denoted by an asterisk in the source name. 

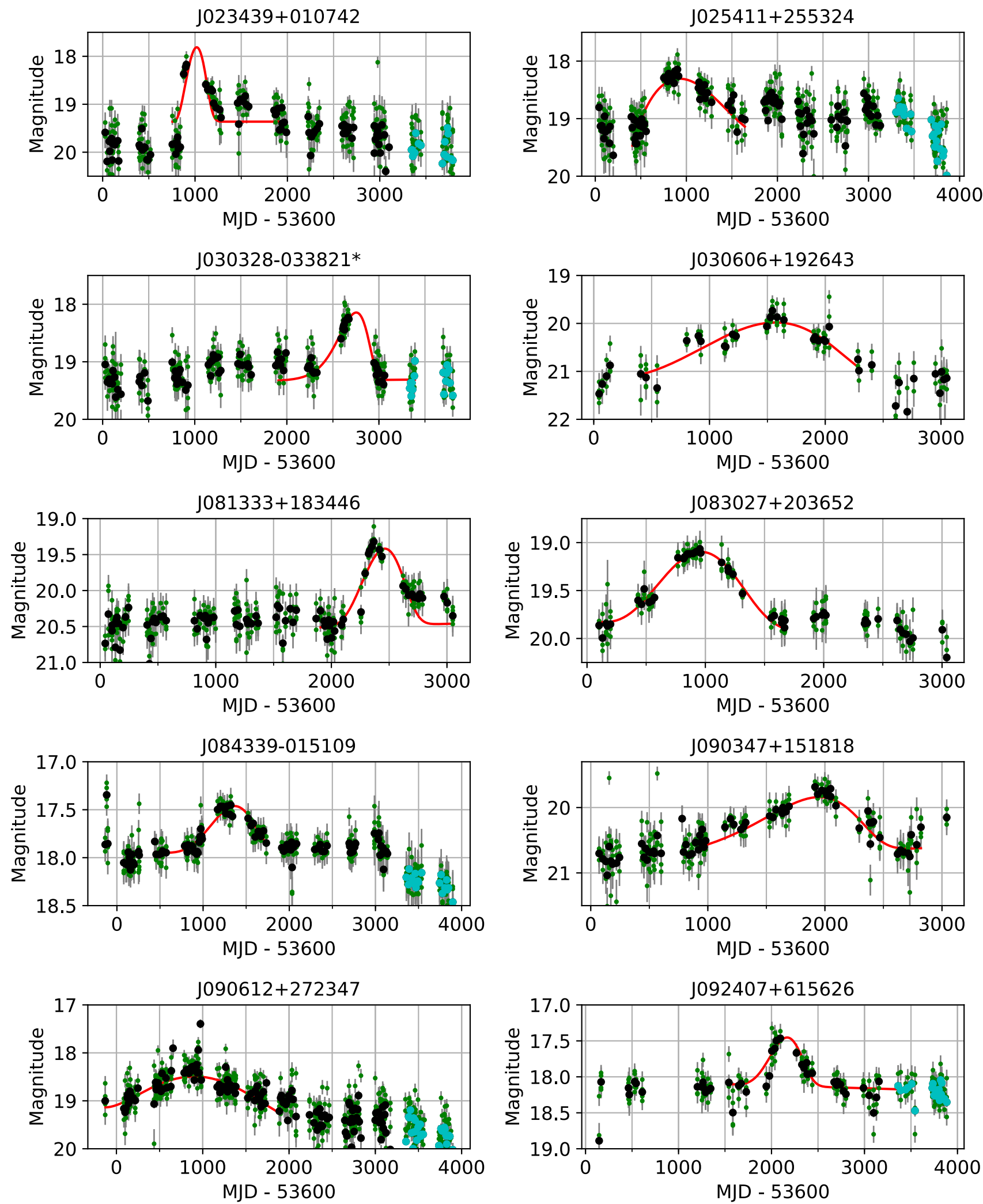

Figure 13 - continued 

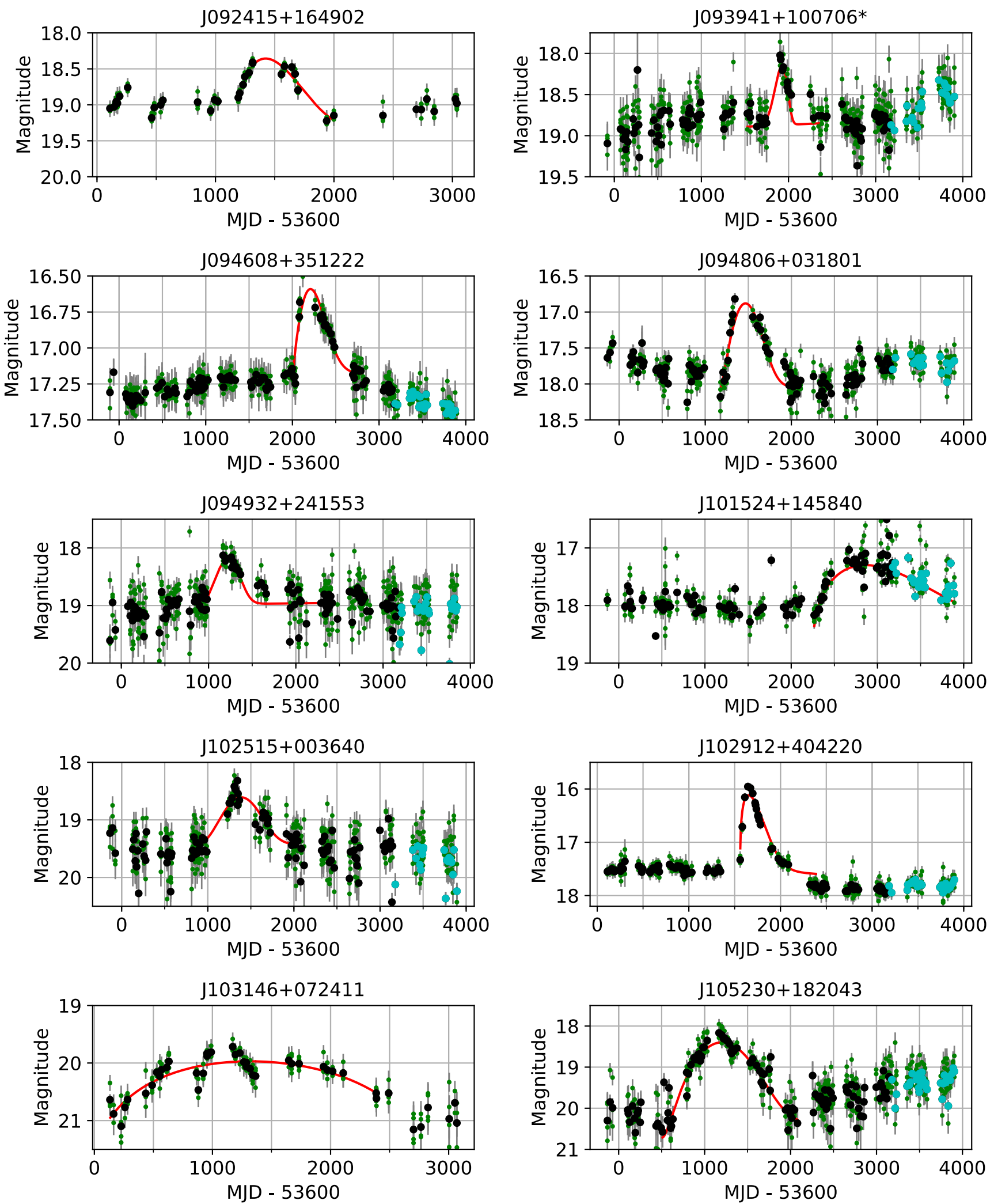

Figure 13 - continued 

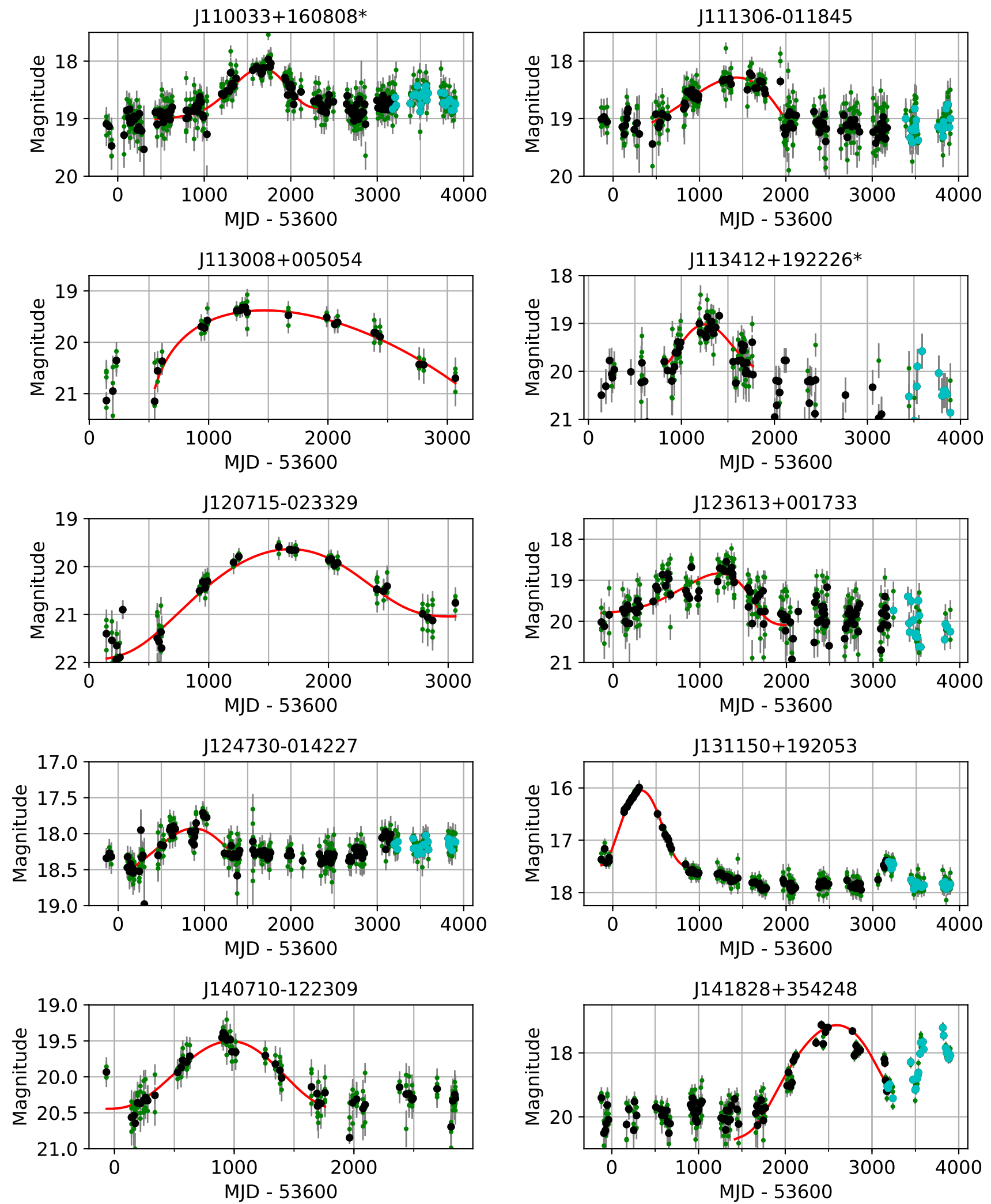

Figure 13 - continued 

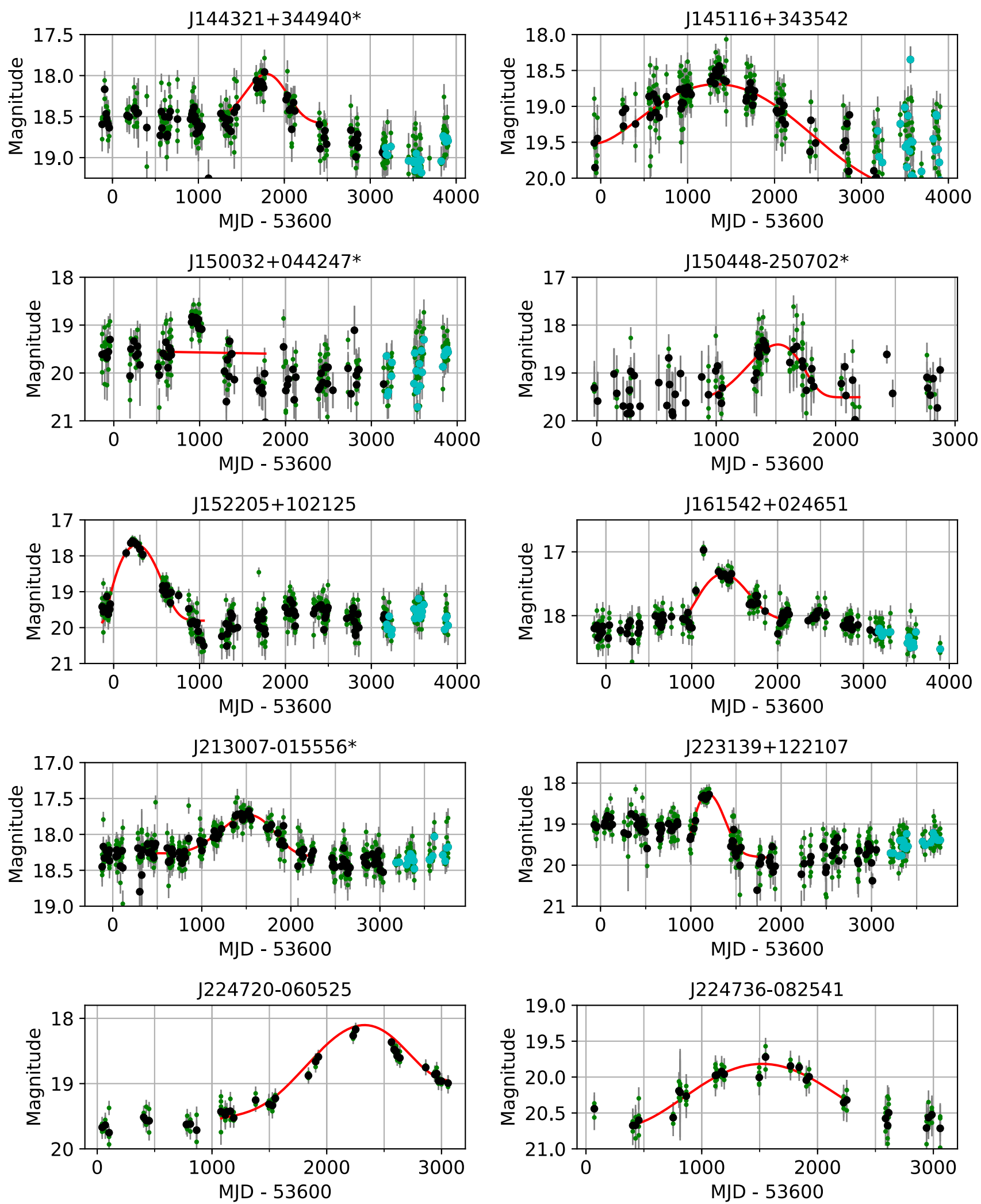

Figure 13 - continued 

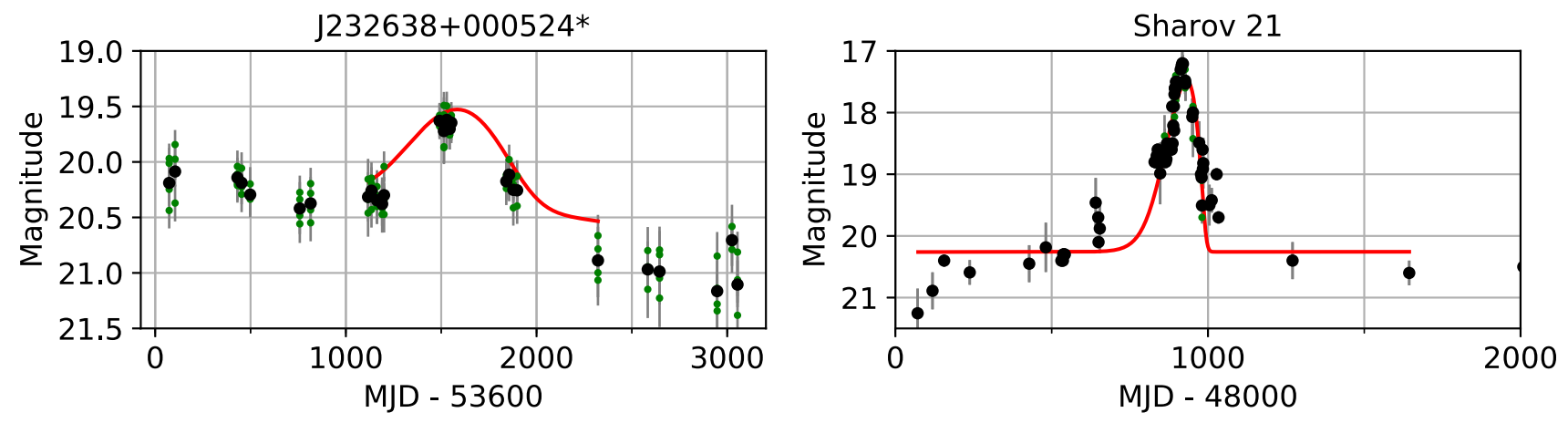

Figure 13 - continued

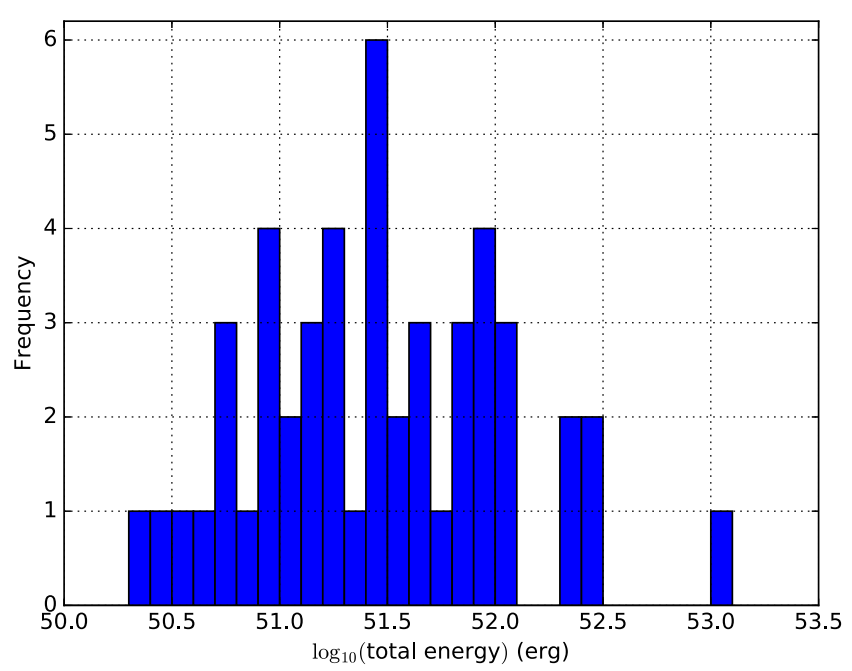

Figure 14. Frequency distribution for total energy output by the flare.

Table 3. The prior distributions used for simulating (and fitting) singlepoint single/double-lens models.

\begin{tabular}{lcc}
\hline Parameter & Symbol & Distribution \\
\hline Source redshift & $z_{\mathrm{s}}$ & CRTS+XDQSO with $V<19$ \\
Background flux & $F_{v, g s}$ & CRTS + XDQSO with $V<19$ \\
Lens redshift & $z_{\mathrm{l}}$ & Uniform over $\left[0.0, z_{\mathrm{s}}\right]$ \\
Lens mass & $m_{\mathrm{l}}$ & Mass-weighted Chabrier (2003) IMF \\
Transverse velocity & $v_{\mathrm{t}}$ & $300 \mathrm{~km} \mathrm{~s}^{-1}[0,400]$ \\
Minimum impact parameter & $u_{0}$ & Uniform over [0.063, 0.63] \\
Time sampling & - & CRTS first-order time difference \\
Second mass time offset & - & Uniform over $[-500,500]$ \\
\hline
\end{tabular}

multiplicative magnification (Meusinger et al. 2010). The resulting distribution is essentially the same as that from the single-point single-lens model and so we do not consider it any further here.

Collectively, the single-point single-lens model is not a good match to the flare candidates we have identified: our sources consistently have larger scale and smaller shape Weibull parameters, indicating more broader and less peaked flares. We note as well that L16 found that only three of their objects showed behaviour that was consistent with a simple point-lens point source system. However, there are a number of the flare candidates whose Weibull parametrization overlaps with that of the single-point single-lens model. We have therefore determined best-fitting single-point single-lens models for each flare using MCMC (via the PYTHON emcee package; ForemanMackey et al. 2013) and the priors described above. For the lens redshift, we assume a uniform prior over the range $\left[0, z_{\mathrm{s}}\right]$, where $z_{\mathrm{s}}$ is the source redshift. When the source redshift is unknown, we assume a fiducial value of $z=1.309$, which is the median redshift of the CRTS data set. Table 4 gives the best-fitting parameters for the 11 candidates that are well fitted $\left(\chi_{\text {red }}^{2}<1.8\right)$ by a single-point single-lens model (see Fig. 17).

In Section 4, we compared the DRW model parameter values for light curves with and without the identified flares. Bruce et al. (2017) performed a similar analysis on two of the L16 lensing candidates, finding that DRW model parameters were atypical for quasars for the observed data but more typical after subtraction of a microlensing model. We have also checked the effect on DRW model parameter values of subtracting the best-fitting single-point single-lens models from the 11 lensed candidates. We find that the residual light curves of nine objects $\left(\chi_{\text {red }}^{2}<1.55\right)$ are more consistent with a DRW variability. However, two objects (with $\chi_{\text {red }}^{2}>1.55$ ) show little difference in their DRW parameter values between the original light curve and the lens model subtracted curve. This supports the microlensing hypothesis for the nine sources but we defer a fuller analysis of them and their spectra to a future paper.

L16 identified CRTS transient data for 16 of their sources (four of which are AGNs); however, CRTS archival data are available for 58 of their AGNs, including 14 of the 15 identified in Stripe 82. Of these, we have identified nine that meet our criteria for a major AGN flare [three of these are also included in Bruce et al. (2017) as lensed sources]. It should be noted that L16 actually distinguish between four categories of AGN light-curve shape: rising, falling, peaked and complex. The rising and falling types are more consistent with behaviour associated with changing-look quasars rather an outburst event and we defer further discussion of this to a subsequent paper. The Weibull characterizations for the nine events (see Table 5) have only partial overlap with the distribution from simulated lens flares, suggesting again that lensing is the physical explanation for the flare in only some of the sources and that a number of different physical mechanisms are contributing overall to these phenomena.

\subsection{Superluminous supernovae}

During the past decade dozens of superluminous supernova have been discovered by wide-field transient surveys such as CRTS, PTF and PS1. Supernovae are defined as superluminous (SLSN) when they reach $M_{V}<-21$ (Drake et al. 2010; Gal-Yam 2012). The most luminous SLSNe observed have $M_{V} \sim-22.5$ (Gal-Yam 2012). In contrast, regular supernovae typically peak in the range $-17<M_{V}$ $<-20$ (Richardson et al. 2002).

SLSNe are generally divided into the hydrogen-poor (SLSN-I) and hydrogen-rich (SLSN-II) types (Gal-Yam 2012). The origin of 

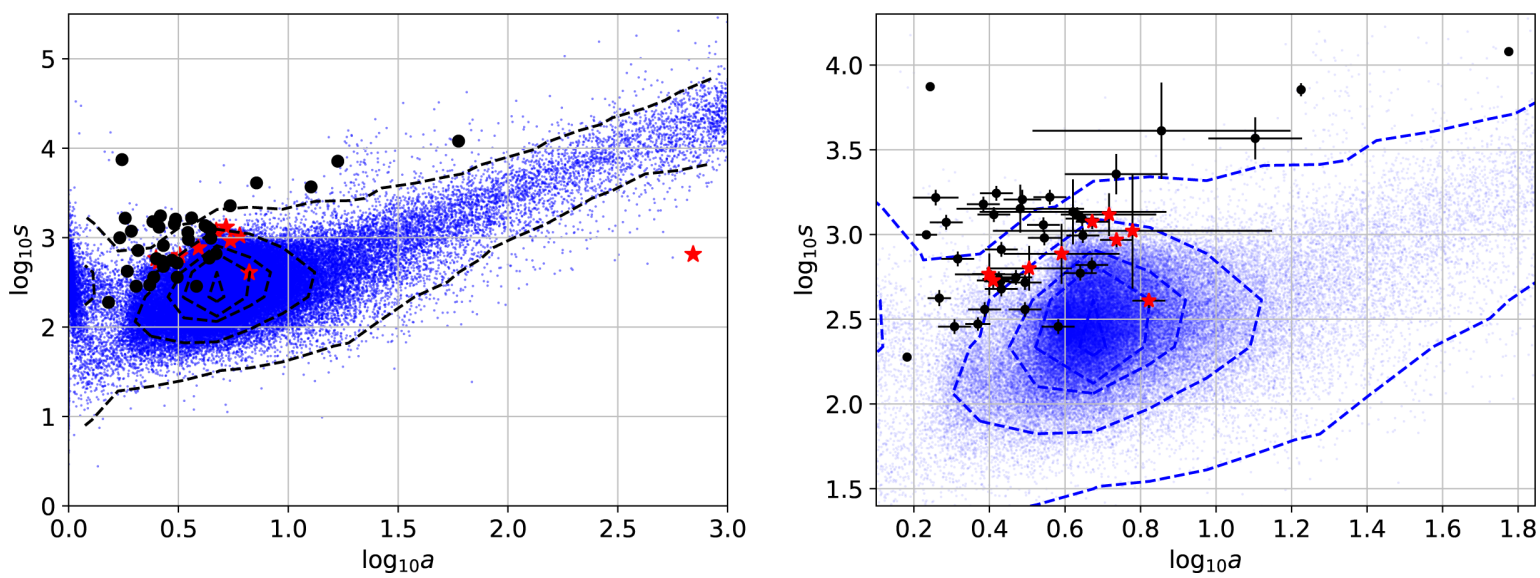

Figure 15. The distribution of the Weibull shape $(a)$ and scale $(s)$ parameters for the flare candidates (black), and simulated flares (blue) according to a single-point single-lens model. The right plot shows an enlargement of the main simulated distribution together with $1 \sigma$ confidence limits on the parameters. Dashed contour lines are shown for 20 per cent density increments. Red stars indicate the lensing candidates.

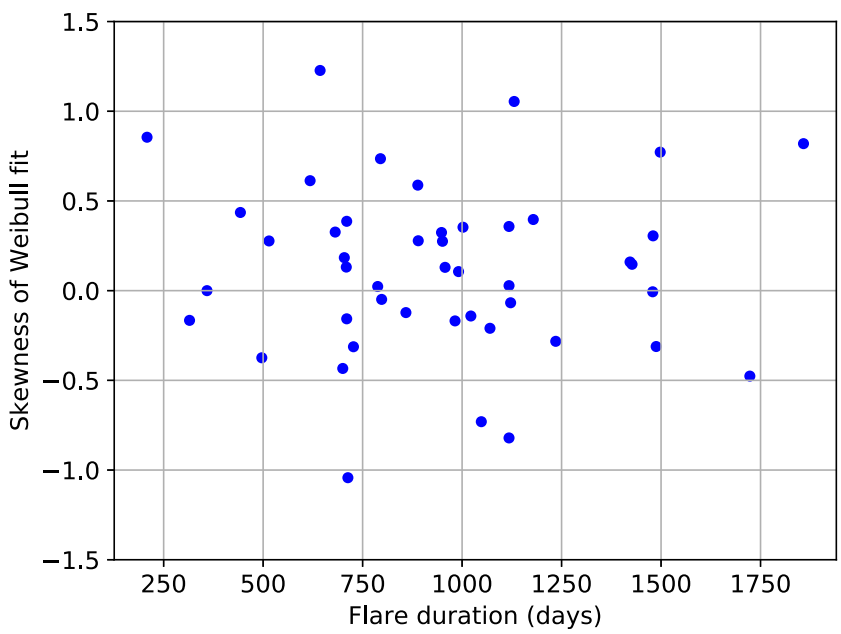

Figure 16. The skewness of the Weibull fit to the flare candidates against the duration of the flare.

Table 4. The best-fitting MCMC parameters for single-point single-lens models

\begin{tabular}{lccccccc}
\hline ID & $z$ & $\begin{array}{c}m_{\mathrm{l}} \\
\left(\mathrm{M}_{\odot}\right)\end{array}$ & $\begin{array}{c}v_{\mathrm{t}} \\
\left(\mathrm{km} \mathrm{s}^{-1}\right)\end{array}$ & $\begin{array}{c}t_{0} \\
(\text { day })\end{array}$ & $z_{\mathrm{l}}$ & $u_{0}$ & $\chi_{\text {red }}^{2}$ \\
\hline $\mathrm{J} 093941+100706$ & 0.328 & 0.69 & 540 & 701 & 0.16 & 0.80 & 0.771 \\
$\mathrm{~J} 150448-250702$ & - & 2.09 & 380 & 582 & 0.61 & 0.47 & 0.894 \\
$\mathrm{~J} 213007-015556$ & 0.290 & 0.66 & 130 & 1125 & 0.13 & 0.70 & 1.272 \\
$\mathrm{~J} 144321+344940$ & 0.749 & 1.08 & 300 & 557 & 0.32 & 0.66 & 1.308 \\
$\mathrm{~J} 110033+160808$ & $(1.090)$ & 0.79 & 210 & 1205 & 0.44 & 0.59 & 1.311 \\
$\mathrm{~J} 030328-033821$ & 0.703 & 12.2 & 609 & 778 & 0.415 & 0.522 & 1.324 \\
$\mathrm{~J} 004133+212841$ & 0.343 & 1.33 & 480 & 1066 & 0.17 & 0.84 & 1.328 \\
$\mathrm{~J} 113412+192226$ & 0.843 & 7.12 & 597 & 476 & 0.365 & 0.414 & 1.442 \\
$\mathrm{~J} 232638+000524$ & 1.031 & 1.38 & 470 & 475 & 0.43 & 0.52 & 1.545 \\
$\mathrm{~J} 010234+050853$ & 1.432 & 8.59 & 315 & 542 & 1.073 & 0.554 & 1.595 \\
$\mathrm{~J} 150032+044247$ & 0.971 & 5.67 & 295 & 412 & 0.480 & 0.480 & 1.672 \\
\hline
\end{tabular}

SLSN-I events is not yet certain but in at least some cases is likely to be due to Wolf-Rayet stars (Taddia et al. 2016). In contrast, SLSNe-II have been attributed to more luminous versions of Type IIn supernova, which themselves are believed to be due to the end stages of luminous blue variables (LBVs) with massive circumstellar matter (CSM) envelopes. Both types of SLSNe have been measured to emit more than $10^{51}$ erg of energy (Smith et al. 2007; Drake et al. 2010; Quimby et al. 2011; Rest et al. 2011) and are thus within the range of almost all the flares shown here. Furthermore, examples of SLSNe have been discovered up to redshifts of $z=3.9$ (Cooke et al. 2012). So the distances to these flaring sources are not exceptional.

One of the clearest signatures of a supernova is a smooth rising light curve followed by a typically much longer decline. The time-scale of the rise varies between supernova types. For example, Type Ia supernovae have rise times of only 2 weeks, whereas Type IIn can take months. Nevertheless, the overall shape of the rising curves is driven by expansion and has long been known to be similar for differing types of supernovae (Wheeler \& Harkness 1990). Our fits to many of the flares presented appear consistent with the asymmetric shapes of supernova light curves. However, the average time-scale of the flares is $\sim 900 \mathrm{~d}$. This is inconsistent with those observed for either regular supernovae or SLSN-I (which generally last $\lesssim 200$ rest-frame days). On the other hand, Type IIn supernovae and the related SLSN-II can last for years (Mahabal et al. 2009; Fox et al. 2015). Nevertheless, although the total energies of the flares are within the range of SLSNe-II, two-thirds of the flares have peak absolute magnitudes brighter than $M_{V}=-23$. The combination of long time-scales and high peak magnitudes suggests that if the flares are due to SLSNe-II, they would have to be an extreme tail.

For SLSNe-II the time-scales of the event depends on both the extent and configuration of the circumstellar material (CSM) environment in which they reside (Chatzopoulos et al. 2013). It is possible that star formation within the AGN disc could lead to the production of massive stars (Levin 2007). The short lifetime of such stars in turn is expected to produce Type II supernovae. If the ejecta from these events could interact with both the CSM from their own outbursts and the gas from the AGN disc, the events may be more luminous and longer lived than historical SN that have overwhelmingly been observed away from the cores of galaxies in order to avoid AGN.

One possible example of an SLSN-II associated with an AGN is CSS100217:102913+404220 (Drake et al. 2011). However, the presence of an AGN, combined with the similarity of AGN and type IIn spectra, has meant that the event could not be firmly identified 

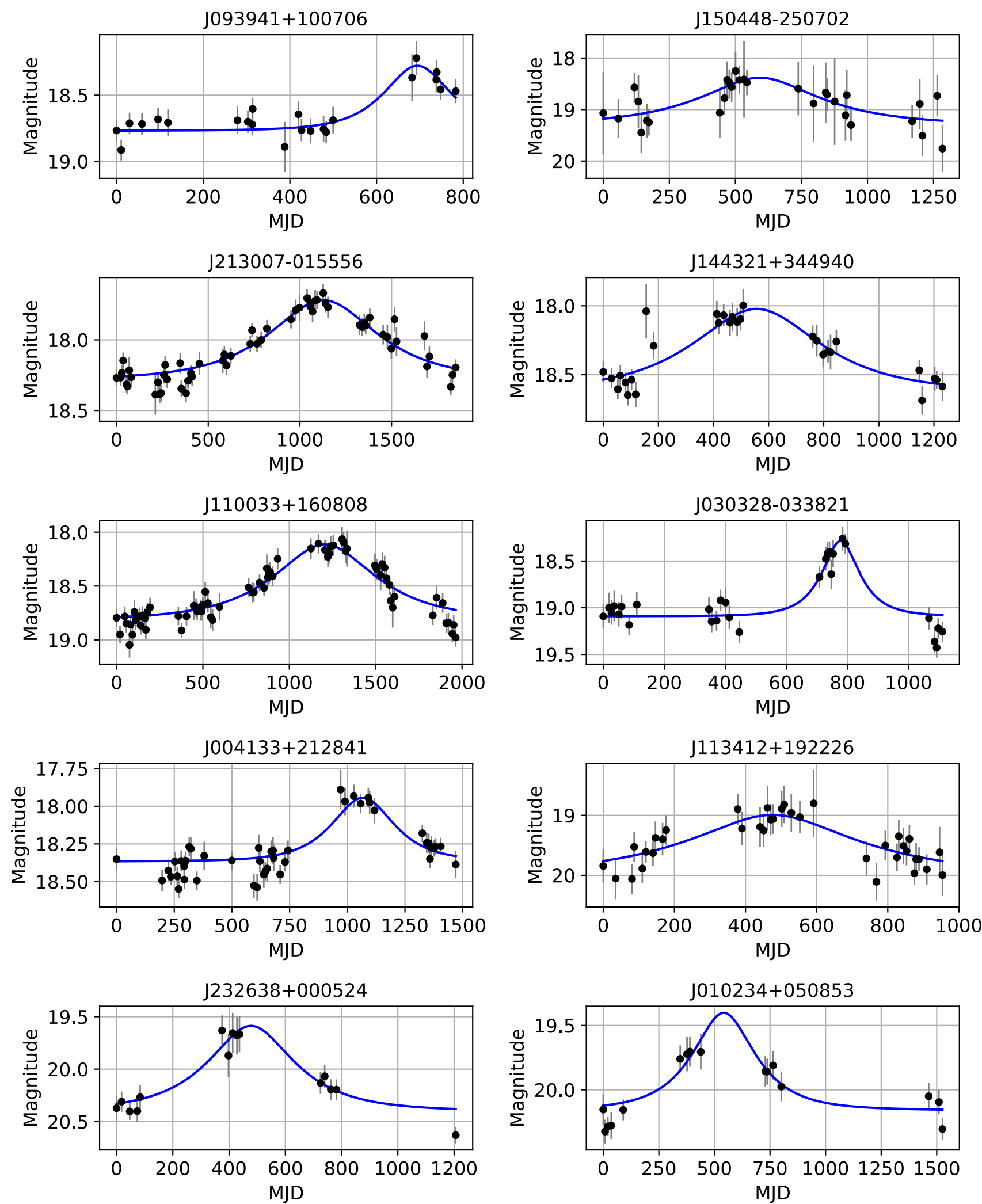

Figure 17. Best-fitting single-point single-lens sources in order of increased reduced chi-square.

as an SLSN. Overall, it seems very unlikely that all the flares we observe could be due to SLSNe-II. For example, many of the flares have longer rise times than declines. Such events are yet to be observed among known supernovae. The recent discovery that the putative most luminous SLSN-I (ASASSN-15lh; Dong et al. 2016) is more likely a TDE than a supernova (Brown et al. 2016; Leloudas et al. 2016) also suggests that very bright flares need not be due to SLSNe. 


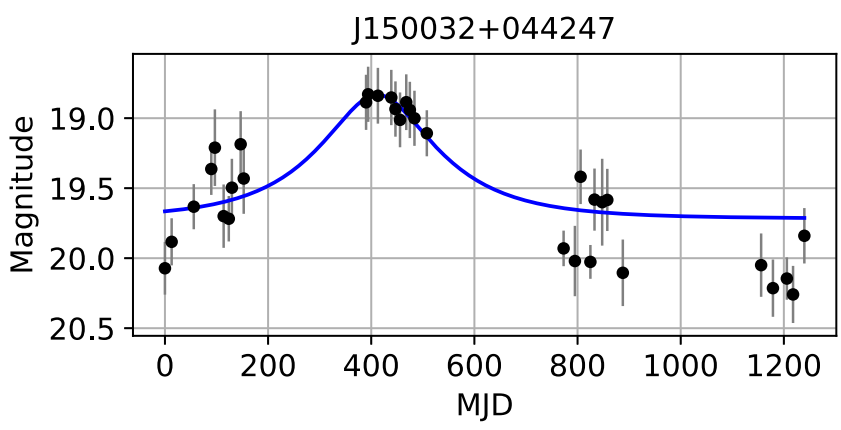

Figure 17 - continued

\subsection{Stellar mass binary black hole merger}

An intriguing potential cause of the observed AGN flaring is a stellar mass binary black hole merger within the dense medium of an AGN accretion disc. The Laser Interferometer Gravitional-Wave Observatory (LIGO) has recently reported the exciting detection of gravitational waves from multiple double stellar mass black hole binary systems, the first due to the merger of a $36_{-4}^{+5} \mathrm{M}_{\odot}$ and 29 $\pm 4 \mathrm{M}_{\odot}$ black holes (GW150914; Abbott et al. 2016a), and the second due to the merger of a $14.2_{-3.7}^{+8.3} \mathrm{M}_{\odot}$ and $7.5 \pm 2.3 \mathrm{M}_{\odot}$ black holes (GW151226; Abbott et al. 2016b). Stellar mass black holes are expected to sink towards the nuclei of galaxies due to dynamical friction with stars. Some of these stellar mass black holes will have formed in binaries, while others will form due to dynamical interactions in dense stellar systems, such as found in galactic nuclei. For galaxies hosting AGNs, many of these black hole binary systems will migrate into the associated accretion disc (McKernan et al. 2017).

Bartos et al. (2017) investigate the time-scales for both the orbital alignment of stellar mass black hole orbits with the accretion disc and the accelerated merger time-scale of stellar mass black hole binaries within an accretion disc. For their fiducial model of a $75 \mathrm{M}_{\odot}$ black hole (which is expected to be essentially equivalent to black hole binary system totalling that mass) and a $10^{6} \mathrm{M}_{\odot}$ central supermassive black hole, they find that a significant fraction ( $\sim 12$ per cent) of stellar mass black holes/black hole binary systems will align themselves with the accretion disc within $10^{7} \mathrm{yr}$. This fraction rises to $\sim 43$ per cent within $10^{8} \mathrm{yr}$. In the dense medium of an accretion disc, the binaries will then merge at an accelerated pace as compared to isolated stellar mass black hole binaries, with mergers expected within $\sim 10^{6} \mathrm{yr}$, first primarily driven by dynamical friction (at early stages) and later due to gravitational radiation (in the final stages).
In the accretion disc, the black holes are also expected to accrete gas from the disc at levels well above the Eddington rate, producing significant high-energy emission. While Bartos et al. (2017) primarily investigate the gravitational wave and high-energy electromagnetic signatures of such events, we make the speculative suggestion that these optical AGN flares could be due to secondary emission related to stellar mass black hole binaries in the dense environment of an accretion disc, either during their pre-merger, super-Eddington accretion stages or, perhaps, they are related to the black hole merger event, though we note that the latter seems less likely given the discrepancy in the time-scales.

It is also possible that the flaring might be related to a single stellar mass black hole migrating through an AGN accretion disc due to torques from gas that is corotating and at (inner and outer) Lindblad resonances (McKernan et al. 2014). Although the migration is a long-term $\left(\sim 10^{6} \mathrm{yr}\right)$ process, a sufficiently massive migrator (compared to the comoving disc gas) could open a gap in the gas disc that would act as a migration trap. A stalled migrator at such a trap may generate a tidal bulge in the gas exterior to its orbit, if the disc is relatively viscous and gas continues to flow inwards. If the bulge suddenly collapses, it might generate a flare. A plunging low angular momentum retrograde orbiter would also generate a flare as it ploughs through the inner disc by dropping low angular momentum gas on to the central black hole.

\subsection{Slow TDEs}

A typical TDE shows a fast rise to a peak luminosity of $\sim 10^{44} \mathrm{erg}$ followed by a decay following $t^{-5 / 3}$ with a time-scale to consume half the material of $t_{1 / 2} \sim 120 \mathrm{~d}$. Such events are also more frequent around lower mass black holes $\left(\sim 10^{6-7} \mathrm{M}_{\odot}\right)$ because of steeper force gradients. Although these characteristics are different from the events described here, Guillochon \& Ramirez-Ruiz (2015) have shown that relativistic precession arising from black hole spin can prevent the debris stream from the TDE from self-intersecting until after many windings. This introduces a delay, possibly of several years, before the flare becomes observable and a shallower powerlaw decline closer to $t^{-1}$ for lower mass black holes. Such TDEs will be sub-Eddington at the peak and so will have been missed in current searches.

We have determined the best-fitting decay profile for each flare via a Thiel-Sen fit in log-space to the flare flux (see Table 6). We find eight candidates with flares characterized by a decaying exponent between -1 and -1.7 , which represents the expected range. One of these (J213007-015556) is also a lensing candidate that we exclude as a TDE candidate as the flare profile is too symmetrical. Only two of the remaining seven sources (J005448+225123 and

Table 5. The list of AGN flare candidates from Lawrence et al (2016) meeting our criteria.

\begin{tabular}{|c|c|c|c|c|c|c|c|c|}
\hline ID & CRTS ID & $V_{\text {med }}$ & $z$ & $\begin{array}{l}\Delta T \\
\text { (d) }\end{array}$ & $\begin{array}{c}a m p_{\max } \\
(\mathrm{mag})\end{array}$ & $a$ & $s$ & $\begin{array}{l}\text { Total energy } \\
\text { (erg) }\end{array}$ \\
\hline $\mathrm{J} 025633+370712$ & 1138013014735 & 19.70 & 0.000 & 635 & 1.49 & 2.52 & 364 & $4.20 \times 10^{37}$ \\
\hline $\mathrm{J} 083714+260932$ & 1126042018137 & 20.07 & 0.000 & 683 & 1.65 & 1.03 & 311 & $1.89 \times 10^{40}$ \\
\hline J090514+503628 & 1149033050415 & 19.91 & 1.290 & 438 & 0.70 & 1.08 & 6110 & $3.74 \times 10^{51}$ \\
\hline J094511+174544 & 2117130011595 & 20.58 & 0.758 & 1172 & 0.85 & 1190 & 480000 & $1.84 \times 10^{51}$ \\
\hline $\mathrm{J} 103837+021119$ & 1101057044911 & 19.62 & 0.620 & 735 & 1.06 & 7.71 & 2530 & $1.24 \times 10^{51}$ \\
\hline $\mathrm{J} 104617+553336$ & 1155035041492 & 20.22 & 0.000 & 470 & 1.70 & 2.87 & 268 & $8.52 \times 10^{41}$ \\
\hline $\mathrm{J} 105501+330002$ & 1132051039346 & 19.29 & 0.417 & 833 & 1.04 & 3.96 & 722 & $7.46 \times 10^{44}$ \\
\hline $\mathrm{J} 142232+014026$ & 1101077043951 & 19.45 & 1.079 & 735 & 0.67 & 1.59 & 1670 & $2.21 \times 10^{51}$ \\
\hline $\mathrm{J} 150210+230915$ & 1123074014056 & 19.95 & 0.630 & 544 & 1.64 & 3.19 & 1690 & $6.67 \times 10^{48}$ \\
\hline
\end{tabular}


Table 6. The list of AGN flare candidates. Sources for which we have obtained a spectroscopic redshift are marked with an asterisk '*'; photometric redshifts are quoted in parentheses. $\Delta T$ is the observed time span of the light curve. $a$ and $s$ are the fitted Weibull parameters. Decay is the best-fitting exponent to the flare profile. Note that sources without a redshift do not have a redshift-corrected total energy value.

\begin{tabular}{|c|c|c|c|c|c|c|c|c|c|c|c|c|}
\hline ID & CRTS ID & RA & Dec. & $V_{\text {med }}$ & $z$ & $\begin{array}{l}\Delta T \\
\text { (d) }\end{array}$ & $\begin{array}{c}a m p p_{\max } \\
(\mathrm{mag})\end{array}$ & $a$ & $s$ & $\begin{array}{c}\text { Peak. abs. } \\
\text { mag. }\end{array}$ & $\begin{array}{l}\text { Total energy } \\
\text { (erg) }\end{array}$ & Decay \\
\hline $00727-132644$ & 12001011298 & 0727.65 & 132644.16 & 8.86 & $0.699 *$ & 2891 & 1.03 & 3.07 & 1610 & -23.5 & $3.04 \times 10^{51}$ & -1.3 \\
\hline J002237+000519 & 2100006002614 & 002237.91 & +000519.14 & 20.47 & 1.373 & 2996 & 1.15 & 2.42 & 1510 & -23.5 & $3.09 \times 10^{51}$ & -1.2 \\
\hline J004133+212841 & 1121004039565 & 004133.26 & +212841.52 & 18.37 & 0.343 & 3031 & 0.89 & 5.45 & 929 & -22.0 & $5.90 \times 10^{50}$ & -3.7 \\
\hline $\mathrm{J} 005448+225123$ & 1123005006362 & 005448.53 & +225123.76 & 17.76 & $0.744 *$ & 1954 & 1.02 & 2.69 & 529 & -25.3 & $1.22 \times 10^{52}$ & -1.0 \\
\hline $\mathrm{J} 010032+042408$ & 2104014001615 & 010032.04 & +042408.42 & 19.31 & 0.721 & 3024 & 0.88 & 12.7 & 3690 & & $3.10 \times 10^{51}$ & -1.6 \\
\hline $\mathrm{J} 012612+113016$ & 2111020015465 & 012612.34 & +113016.20 & 19.82 & 0.800 & 2942 & 1.18 & 7.17 & 4090 & -23.5 & $3.56 \times 10^{51}$ & -1.8 \\
\hline J022014-072859 & 1007013018204 & 022014.57 & -072859.34 & 17.03 & 0.213 & 2922 & 0.78 & 59.6 & 12000 & -22.0 & $1.14 \times 10^{51}$ & -0.3 \\
\hline $\mathrm{J} 023439+010742$ & 1101014022249 & 023439.07 & +010742.67 & 19.50 & 0.277 & 2881 & 1.96 & 3.82 & 286 & -21.5 & $5.22 \times 10^{50}$ & - \\
\hline $\mathrm{J} 025411+255324$ & 1126015012060 & 025411.02 & +255324.72 & 18.84 & 0.331 & 2877 & 1.57 & 2.07 & 718 & -2 & $8.13 \times 10^{50}$ & -1.9 \\
\hline J030328-033821 & 1004017038032 & 030328.63 & -033821.59 & 19.09 & $0.703 *$ & 2963 & 1.30 & $>10^{5}$ & $>10^{5}$ & -24.0 & $2.10 \times 10^{51}$ & -3.9 \\
\hline J030606+192643 & 2118041030282 & 030606.67 & +192643.08 & 20.55 & $0.522 *$ & 2939 & 1.85 & 5.44 & 2270 & -22.5 & $1.66 \times 10^{51}$ & -2.5 \\
\hline J090612+272347 & 1126045043734 & 090612.24 & +272347.40 & 18.74 & $0.920^{*}$ & 2211 & 1.66 & 2.58 & 1310 & -21.5 & $6.46 \times$ & -1.1 \\
\hline J092407+615626 & 1160026056310 & 092407.68 & +615626.52 & 18.10 & 0.205 & 2653 & 0 & 4.69 & 661 & - & $3.34 \times$ & -0.2 \\
\hline J092415+164902 & 2116126012459 & 092415.36 & +164902.28 & 18.93 & 0.352 & 2927 & 0. & 1.85 & 421 & - & $5.35 \times 10^{50}$ & -2.8 \\
\hline J093941+100706 & 1109052036713 & 093941.04 & +100706.60 & 18.80 & 0.328 & 286 & & 6.64 & & & $2.51 \times 10^{50}$ & -0.4 \\
\hline J094608+351222 & 1135044028613 & 094608.40 & +351222.68 & 17.19 & 0.119 & 2954 & 0. & 2.03 & 286 & -2 & $3.14 \times 10^{50}$ & -2.8 \\
\hline J094806+031801 & 1104053011095 & 094806.48 & +031801.44 & 17.83 & 0.207 & 2961 & 1 & 2.44 & 361 & -22.0 & $8.82 \times 10^{50}$ & -3.7 \\
\hline J094932+241553 & 1123049035019 & 094932.64 & +241553.28 & 18.79 & 1.123 & 2977 & 1 & 3.12 & 361 & -2 & $8.09 \times 10^{51}$ & - \\
\hline $\mathrm{J} 101524+145840$ & 1115054017365 & 101524.72 & +145840.80 & 17.97 & 1.102 & 3122 & 2. & 1.71 & 996 & -2 & $2.60 \times 10^{52}$ & -2.3 \\
\hline $\mathrm{J} 102515+003640$ & 1101056013653 & 102515.36 & +003640.79 & 19.37 & 0.817 & 2492 & 1. & 2.95 & 558 & -24.0 & $2.90 \times 10^{51}$ & -2.2 \\
\hline $\mathrm{J} 102912+404220$ & 1140044024955 & 102912.48 & +404220.16 & 17.50 & 0.147 & 2739 & & 1.52 & & & $8.25 \times 10^{50}$ & -4.0 \\
\hline $\mathrm{J} 103146+072411$ & 2107146024798 & 1031 & +072411.30 & 20.16 & $(1.0$ & 2936 & 1. & 1.75 & 74 & -2 & $10^{51}$ & -0.8 \\
\hline $\mathrm{J} 105230+182043$ & 1118056030240 & 105230.48 & +182043.08 & 19.46 & 0.693 & 3127 & 2.45 & 2.7 & 816 & -2 & 6 & -4.0 \\
\hline J140710-122309 & 2012192015243 & 140710.32 & -122309.24 & 20.18 & 08 & 2914 & 1 & 3.49 & & -2 & $1.30 \times 10^{51}$ & -1.8 \\
\hline $\mathrm{J} 141828+354248$ & 1135064040384 & 141828.56 & +354248.96 & 19.66 & & 2984 & 2.53 & 4.42 & 1240 & & $1.08 \times$ & -10.2 \\
\hline $\mathrm{J} 144321+344940$ & 1135066022284 & 144321.12 & +344940.44 & 18.48 & 0.749 & 2980 & 1.03 & 3.2 & 631 & -24.0 & $3.26 \times 10^{51}$ & -2.1 \\
\hline $\mathrm{J} 145116+343542$ & 1135066016768 & 145116.08 & +343542.36 & 18.90 & $(1.475)$ & 29 & 1.2 & 2.62 & 1750 & -2 & $1.12 \times 10^{52}$ & -1.8 \\
\hline $\mathrm{J} 150032+044247$ & 1104081049591 & 150032.88 & +044247.20 & 19.64 & $(0.971)$ & 2990 & 1. & 697 & 651 & -24.0 & $1.34 \times 10^{51}$ & - \\
\hline $\mathrm{J} 150448-250702$ & 3025103031853 & 150448.96 & -250703.00 & 19.06 & & 2868 & 1.8 & 6.01 & 1050 & -25.8 & $1.73 \times 10^{37}$ & -3.3 \\
\hline $\mathrm{J} 152205+102125$ & 1109082051016 & 152205.04 & +102125.20 & 19.48 & $(0.903)$ & 2987 & 2.51 & 2.7 & 477 & - & $1.14 \times 10^{52}$ & -1.9 \\
\hline $\mathrm{J} 161542+024651$ & 1101087096238 & 161542.72 & +024651.13 & 18.04 & $0.326^{*}$ & 3071 & 1.28 & 2.51 & 582 & -23.0 & $2.90 \times 10^{51}$ & -1.8 \\
\hline $\mathrm{J} 213007-015556$ & 1001115026824 & 213007.92 & -015556.93 & 18.23 & 0.290 & 3111 & 0.86 & 4.7 & 1190 & -22.0 & $1.01 \times 10^{51}$ & -1.7 \\
\hline $\mathrm{J} 223139+122107$ & 1112119025810 & 223139.84 & +122107.92 & 19.27 & $0.603^{*}$ & 3055 & 1.81 & 2.34 & 296 & -23.3 & $4.74 \times 10^{44}$ & -3.6 \\
\hline $\mathrm{J} 224720-060525$ & 2005315009619 & 224720.88 & -060525.87 & 19.26 & $1.669 *$ & 3013 & 1.08 & 4.29 & 1310 & -26.0 & $2.36 \times 10^{52}$ & -3.6 \\
\hline J224736-082541 & 2008314006156 & 224736.96 & -082541.02 & 20.30 & $1.638^{*}$ & 2986 & 1.21 & 3.03 & 1420 & & & -1.3 \\
\hline $\mathrm{J} 232638+000524$ & 2000326023025 & 232638.16 & +000524.65 & 20.32 & 1.031 & 2949 & 1.07 & 3.9 & 769 & -23.3 & $1.91 \times 10^{51}$ & -3.0 \\
\hline Sharov 21 & - & 004457.94 & +412343.90 & 19.2 & 2.109 & 0 & 3.03 & 2190 & $>10^{5}$ & - & $1.39 \times 10^{52}$ & - \\
\hline
\end{tabular}

J010234+050853) have sufficiently short rise times to be considered a viable TDE event(the other five candidates all have a longer rise time than fall time that is not the expected profile). From the spectra of these quasars, we measure $\mathrm{Mg}$ II equivalent widths of $1100 \mathrm{~km} \mathrm{~s}^{-1}$ (J005448+225123) and $4500 \mathrm{~km} \mathrm{~s}^{-1}(\mathrm{~J} 010234+050853)$, respectively, which give virial black hole masses of $\log _{10}\left(\mathrm{M} / \mathrm{M}_{\odot}\right)=7.8$ and 8.9 using Kozlowski (2016b). Assuming that the viscous time for the accretion disc is 100 times longer than the orbital period, Guillochon \& Ramirez-Ruiz (2015) find that the majority of events associated with black holes below a fiducial mass of $\log _{10}\left(\mathrm{M} / \mathrm{M}_{\odot}\right)$ $=7.0$ are slowed. A longer viscous time leads to a higher fiducial mass and more slow TDEs around higher mass black holes. The 
less massive of our two candidates, J005448+225134, has the shallower decay slope and longer event duration but further modelling is required to see if slow TDEs are a viable explanation for some of these events.

\section{CONCLUSIONS}

We have identified 51 quasars that over the past decade have exhibited a major flaring event that is statistically distinct from their expected stochastic variability. The event typically lasts about $900 \mathrm{~d}$ (in the observed frame) and has a median peak amplitude of $\Delta m$ $=1.25 \mathrm{mag}$. The flares have one of three distinct profiles: symmetric, fast rise exponential decay, and slow rise, fast decay. L16 have proposed that many such events are attributable to microlensing. A single-point single-lens model provides a good description for nine of the flares but we suggest that the rest are due to some form of explosive stellar activity in the accretion disc: a superluminous supernova, a (slowed) TDE, or even a merger event. Further modelling, both of more complex lensing geometries and stellar-initiated activity within the accretion disc of an AGN, will help to understand these events.

Follow-up observations, both spectroscopic and multiwavelength, would also help to discriminate between different models. The long baseline of these events means that there may be serendipitous observations in existing archives and we will consider this in a subsequent paper. The increasing number of sky surveys and sky coverage per night also means that more of these events should be discovered in future. We estimate the rate of a $\Delta m=1 \mathrm{mag}$ event with a lifetime of $\sim 1000 \mathrm{~d}$ in the AGN population to be $\sim 10^{-5} \mathrm{yr}^{-1} \mathrm{sr}^{-1}$. A statistically useful sample should therefore be feasible within the first few years of LSST.

Although these events may offer more insight into the structure of the accretion disc, e.g. constraints on the size of particular regions from microlensing, they seem to be a distinct class of phenomenon from the more general variability seen in AGNs. They are true outliers rather than representing the tail of any distribution. One possibility, however, is that these types of explosive events seed more general AGN variability by creating matter streams, shock fronts and inhomogeneities in the (inner) accretion disc. This would then support the type of variability mechanisms proposed by Aretxaga, Cid Fernandes \& Terlevich (1997) and Torricelli-Ciamponi et al. (2000). Again, further modelling is needed to see whether the expected observational signatures match what is actually seen.

\section{ACKNOWLEDGEMENTS}

We thank Hans Meusinger for providing the data for Sharov 21 in the electronic form, Chelsea MacLeod for the predicted cumulative magnitude distributions, Alastair Bruce for clarifying the L16 lens fits, and Barry McKernan for discussions on accretion disc physics. We also thank the anonymous referee for useful comments.

This work was supported in part by the NSF grants AST-1413600 and AST-1518308. The work of DS was carried out at Jet Propulsion Laboratory, California Institute of Technology, under a contract with NASA.

This work made use of the Million Quasars Catalogue.

Funding for SDSS-III has been provided by the Alfred P. Sloan Foundation, the Participating Institutions, the National Science Foundation and the U.S. Department of Energy Office of Science. The SDSS-III web site is http://www.sdss3.org/.

SDSS-III is managed by the Astrophysical Research Consortium for the Participating Institutions of the SDSS-III Collabo- ration including the University of Arizona, the Brazilian Participation Group, Brookhaven National Laboratory, Carnegie Mellon University, University of Florida, the French Participation Group, the German Participation Group, Harvard University, the Instituto de Astrofisica de Canarias, the Michigan State/Notre Dame/JINA Participation Group, Johns Hopkins University, Lawrence Berkeley National Laboratory, Max Planck Institute for Astrophysics, Max Planck Institute for Extraterrestrial Physics, New Mexico State University, New York University, Ohio State University, Pennsylvania State University, University of Portsmouth, Princeton University, the Spanish Participation Group, University of Tokyo, University of Utah, Vanderbilt University, University of Virginia, University of Washington and Yale University.

\section{REFERENCES}

Abbott B. P. et al., 2016a, Phys. Rev. Lett., 116, 061102

Abbott B. P. et al., 2016b, Phys. Rev. Lett., 116, 241103

Aretxaga I., Cid Fernandes R., Terlevich R., 1997, MNRAS, 286, 271

Assef R. J. et al., 2013, ApJ, 772, 26

Bartos I., Kocsis B., Haiman Z., Márka S., 2017, ApJ, 835, 165

Brown P. J. et al., 2016, ApJ, 828, 3

Bruce A. et al., 2017, MNRAS, 467, 1259

Chabrier G., 2003, PASP, 115, 763

Chatzopoulos E., Wheller J. C., Vinko J., Horvath Z. L., Nagy A., 2013, ApJ, 773, 76

Chornock R. et al., 2014, ApJ, 780, 44

Cooke J. et al., 2012, Nature, 491, 228

D’Orazio D. J., Haiman Z., Schiminovich D., 2015, Nature, 525, 351

de Vries W. H., Becker R. H., White R. L., Loomis C., 2005, AJ, 129, 615

DiPompeo M. A., Bovy J., Myers A. D., Lang D., 2015, MNRAS, 452, 3124

Djorgovski S. G. et al., 2012, in Mihara T., Serino M., eds, The First Year of MAXI: Monitoring Variable X-ray Sources. Special Publ. IPCR-127, 263. RIKEN, Tokyo

Dong S. et al., 2016, Science, 351, 257

Drake A. J. et al., 2009, ApJ, 696, 870

Drake A. J. et al., 2010, ApJ, 718, 127

Drake A. J. et al., 2011, ApJ, 735, 106

Drake A. J. et al., 2013, ApJ, 763, 32

Drake A. J. et al., 2014, ApJS, 213, 9

Foreman-Mackey D., Hogg D. W., Lang D., Goodman J., 2013, PASP, 125 , 306

Fox O. D. et al., 2015, MNRAS, 454, 4366

Gal-Yam A., 2012, Science, 337, 927

Gezari S. et al., 2012, Nature, 485, 217

Graham M. J., Djorgovski S. G., Drake A. J., Mahabal A. A., Chang M., Stern D., Donalek C., Glikman E., 2014, MNRAS, 439, 703

Graham M. J. et al., 2015a, Nature, 518, 74

Graham M. J. et al., 2015b, MNRAS, 453, 1562

Guillochon J., Ramirez-Ruiz E., 2015, ApJ, 809, 166

Huenemoerder D. P., Schulz N. S., Testa P., Drake J. J., Osten R. A., Reale F., 2010, ApJ, 723, 1558

Ivezic Z. et al., 2007, AJ, 134, 973

Jarosik N. et al., 2011, ApJS, 192, 15

Kaiser N. et al., 2002, in Tyson J. A., Wolff S., eds, Proc. SPIE Vol. 4836, Survey and Other Telescope Technologies and Discoveries. SPIE, Bellingham, p. 154

Kelly B. C., Bechtold J., Siemiginowska A., 2009, ApJ, 698, 895

Kelly B. C., Becker A. C., Sobolewska M., Siemiginowska A., Uttley P., 2014, ApJ, 788, 33

Kozlowski S., 2016a, ApJ, 826, 118

Kozlowski S., 2016b, MNRAS, 459, 2787

LaMassa S. M. et al., 2015, ApJ, 800, 144

Lang D., Hogg D. W., Schlegel D. J., 2016, AJ, 151, L36

Lawrence A. et al., 2016, MNRAS, 463, 296 (L16)

Leloudas G. et al., 2016, Nat. Astron., 1, L2 
Levin Y., 2007, MNRAS, 374, 515

Liu F. K., Li S., Komossa S., 2014, ApJ, 786, 103

McKernan B., Ford K. E. S., Kocsis B., Lyra W., Winter L. M., 2014, MNRAS, 441, 900

McKernan B. et al., 2017, preprint (arXiv:1702.07818)

MacLeod C. L. et al., 2010, AJ, 721, 1014

MacLeod C. et al., 2012, ApJ, 753, 106

MacLeod C. et al., 2016, ApJ, 457, 389

Mahabal A. A. et al., 2009, Astron. Telegram, 2010

Mahabal A. A. et al., 2011, Bull. Astron. Soc. India, 39, 387

Massaro E., Maselli A., Leto C., Marchegiani P., Perri M., Giommi M., Piranomonte S., 2015, Ap\&SS, 357, 75

Massey P., Gronwall C., 1990, PASP, 102, 1046

Meusinger H. et al., 2010, A\&A, 512, A1

Morganson E. et al., 2014, ApJ, 784, 92

Oke J. B. et al., 1995, PASP, 107, 375

Palaversa L. et al., 2013, AJ, 146, 101

Paris I. et al., 2016, A\&A, 597, A79

Quimby R. M. et al., 2011, Nature, 474, 487

Rafiee A., Pirkola P., Hall P. B., Galati N., Rogerson J., Ameri A., 2016, MNRAS, 459, 2472

Rau A. et al., 2009, PASP, 121, 1334

Rest A. et al., 2011, ApJ, 729, 88

Richardson D., Branch D., Casebeer D., Millard J., Thomas R. C., Baron E., 2002, AJ, 123, 745

Ruan J. J. et al., 2016, ApJ, 826, 188

Schlafly E. F., Finkbeiner D. P., 2011, ApJ, 737, 103

Schlegel D. J., Finkbeiner D. P., Davis M., 1998, ApJ, 500, 525

Sesar B. et al., 2007, AJ, 134, 2236
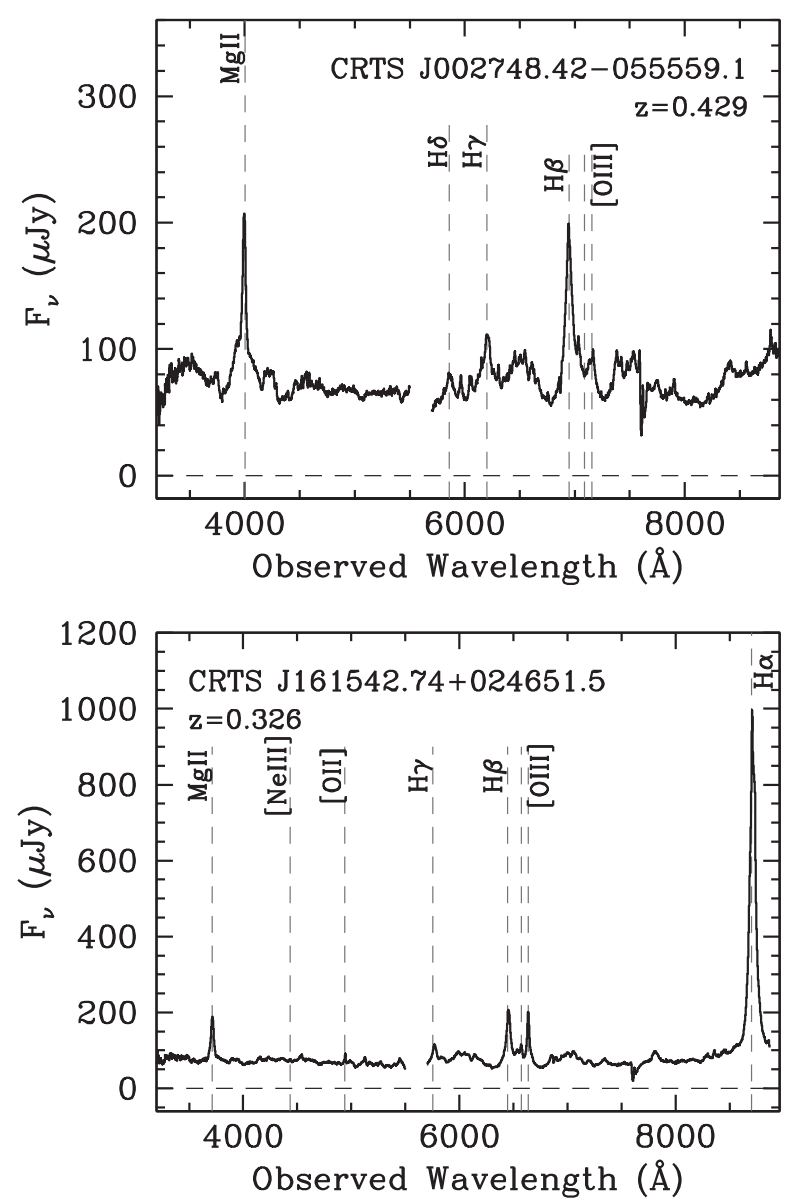

Sesar B., Scott Stuart J., Ivezic Z., Morgan D. P., Becker A. C., Wozniak P., 2011, AJ, 142, 190

Smith N. et al., 2007, ApJ, 666, 1116

Stern D. et al., 2012, ApJ, 753, 30

Stern D. et al., 2017, ApJ, 839, 106

Stone R. P. S, Baldwin J. A., 1983, MNRAS, 204, 347

Stone R. P. S, Baldwin J. A., 1984, MNRAS, 206, 241

Taddia F. et al., 2016, A\&A, 592, 89

Torricelli-Ciamponi G., Foellmi C., Courvoisier T. J.-L., Paltani S., 2000, A\&A, 358, 57

Vaughan S., Uttley P., Markowitz A. G., Huppenkothen D., Middleton M. J., Alston W. N., Scargle J. D., Farr W. M., 2016, MNRAS, 461, 3145

Wheeler J. C., Harkness R. P., 1990, Rep. Prog. Phys., 53, 1467

\section{APPENDIX A: SPECTROSCOPIC OBSERVATIONS OF FLARING CANDIDATES}

A total of 13 of the photometrically identified quasar candidates for which we found evidence of flaring activity did not have published spectroscopy (e.g. spectroscopic redshifts) prior to this work, hampering our ability to fully interpret the results for those sources. Here we briefly describe spectroscopy obtained for these 13 sources, which are indicated with asterisks after their redshifts in Table 6.

We obtained spectroscopic observations at the Palomar and Keck Observatories between 2015 December and 2017 February from our dedicated programme to follow-up CRTS AGN with unusual synoptic properties (see Fig. A1 for examples). Table A1 lists basic
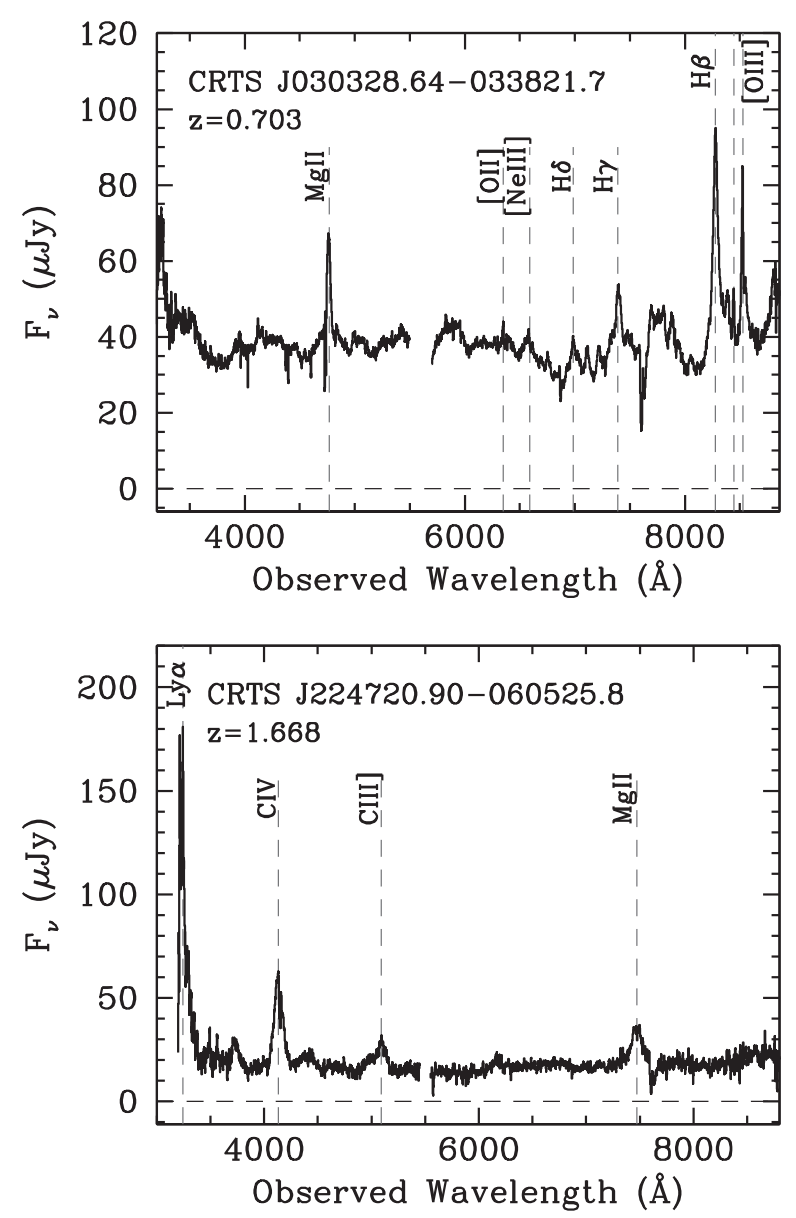

Figure A1. Examples of four optical spectra obtained from our Palomar and Keck follow-up programs. The spectrum of CRTS J224720.90-060525.8 was obtained at Palomar, while the other three spectra were obtained at Keck. Note the strong Fe features in the top panel flanking the $\mathrm{H} \beta$ emission. 
Table A1. Details of the 13 flaring quasars that were observed spectroscopically to derive redshifts.

\begin{tabular}{lccc}
\hline ID & Telescope & UT date & Exp. time (s) \\
\hline J000727-132644 & Keck & 2016 Sept 09 & 900 \\
J002748-055559 & Keck & 2016 Sept 09 & 900 \\
J005448+225123 & Palomar & 2015 Dec 04 & 900 \\
J010234+050853 & Keck & 2016 Sept 09 & 900 \\
J030328-033821 & Keck & 2016 Sept 09 & 900 \\
J030606+192643 & Keck & 2016 Sept 09 & 900 \\
J081333+183446 & Keck & 2016 Dec 29 & 900 \\
J090347+151818 & Palomar & 2017 Feb 25 & 900 \\
J090612+272347 & Palomar & 2017 Feb 25 & 900 \\
J161542+024651 & Keck & 2016 Sept 09 & 900 \\
J223139+122107 & Keck & 2016 Sept 09 & 900 \\
J224720-060525 & Palomar & 2016 Nov 06 & $2 \times 900$ \\
J224736-082541 & Palomar & 2016 Nov 06 & 900 \\
\hline
\end{tabular}

observing details for the 10 flaring quasars observed, including which telescope they were observed with, the date of the observation and the integration time. Palomar observations utilized the dualbeam Double Spectrograph on the 200-inch Hale Telescope, which was configured with the 1.5 arcsec wide slit, the $5500 \AA$ dichroic, the $600 \ell \mathrm{mm}^{-1}$ grating on the blue arm $\left(\lambda_{\text {blaze }}=4000 \AA\right.$; spectral resolving power $R \equiv \lambda / \Delta \lambda \sim 1200$ ), and the $316 \ell \mathrm{mm}^{-1}$ grating on the red $\operatorname{arm}\left(\lambda_{\text {blaze }}=7500 \AA ; R \sim 1800\right)$. Keck observations utilized the dual-beam Low Resolution Imaging Spectrograph (LRIS; Oke et al. 1995) on the Keck I telescope, configured with the 1.5 arcsec wide slit, the $600 \ell \mathrm{mm}^{-1}$ grism on the blue arm $\left(\lambda_{\text {blaze }}=\right.$ $4000 \AA ; R \sim 800)$ and the $400 \ell \mathrm{mm}^{-1}$ grating on the red arm $\left(\lambda_{\text {blaze }}\right.$ $=8500 \AA ; R \sim 1000$ ).

All nights listed in Table A1 were photometric, and we processed the data using standard techniques within IRAF. For all but the 2015 December observation, we calibrated the data using standard stars from Stone \& Baldwin $(1983,1984)$ and Massey \& Gronwall (1990) observed on the same nights using the same instrument configuration; for the 2015 December observation, we used an archival sensitivity function obtained from similar standard stars observed with an identical instrument configuration. For all 13 sources, the spectroscopy revealed quasars with multiple emission features providing robust redshift identifications.

\section{APPENDIX B: STATISTICAL MOMENTS OF THE TRANSLATED WEIBULL DISTRIBUTION}

The translated Weibull distribution is defined as:

$X(p ; a, s)=\left(\frac{a}{s}\right) p^{a-1} \mathrm{e}^{-p^{a}}$

$p=\left(t-t_{0}\right) / s$

in which $a$ is a shape parameter $(a>0)$, the scale (or width) is specified by $s(s>0)$, and the location is given by $t_{0}\left(t_{0} \geq 0\right)$; the independent coordinate is $t\left(t \geq t_{0}\right)$. The first three statistical moments, $\mu, \sigma^{2}, \gamma_{1}$, are:

$E\left(X^{r}\right)=\sum_{i=0}^{r}\left(\begin{array}{l}r \\ i\end{array}\right) t_{0}^{i} s^{r-i} \Gamma\left(1+\frac{r-i}{a}\right)$

$\mu=E(X)=t_{0}+s g_{1}$

$\sigma^{2}=E\left(X^{2}\right)-\mu^{2}=s^{2}\left(g_{2}-g_{1}^{2}\right)$

$\gamma_{1}=\frac{E\left(X^{3}\right)-3 \mu \sigma^{2}-\mu^{3}}{\sigma^{3}}=\frac{g_{3}-3 g_{1} g_{2}+2 g_{1}^{3}}{\left(g_{2}-g_{1}^{2}\right)^{3 / 2}}$

where $g_{i}=\Gamma(1+i / a)$. The skewness of the Weibull distribution is invariant under a location-scale transformation with positive slope: skew $(a+b X)=\operatorname{skew}(X)$, where $b>0$ and changes sign for a negative slope: $\operatorname{skew}(a+b X)=-\operatorname{skew}(X)$ for $b<0$.

This paper has been typeset from a $\mathrm{TE}_{\mathrm{E}} \mathrm{L} \mathrm{LT} \mathrm{T} \mathrm{X}$ file prepared by the author. 